\title{
Explicit $H^{1}$-Estimate for the Solution of the Lamé System with Mixed Boundary Conditions
}

\author{
AIT-AKLI Djamel*and MERAKEB Abdelkader \\ L2CSP, Mouloud Mammeri University Tizi-Ouzou, 15000, Algeria.
}

Received 20 May 2019; Accepted 27 February 2020

\begin{abstract}
In this paper we consider the Lamé system on a polygonal convex domain with mixed boundary conditions of Dirichlet-Neumann type. An explicit $L^{2}$ norm estimate for the gradient of the solution of this problem is established. This leads to an explicit bound of the $H^{1}$ norm of this solution. Note that the obtained upper-bound is not optimal.
\end{abstract}

AMS Subject Classifications: 35J57, 74B05

Chinese Library Classifications: O175.27

Key Words: Lamé system; Korn's inequality; Poincare's inequality; inequality of trace; explicit estimates.

\section{Introduction}

Let $\Omega$ be a bounded open connected subset of $\mathbb{R}^{2}$. The static equilibrium of a deformable structure occupying $\Omega$ is governed by the Lamé linear elasto-static system, see [1]. In this paper, we restrict the study to a convex domain $\Omega$ whose boundary has a polygonal shape that posses $m+1$ edges with $m \geq 2$. We denote $\Gamma=\cup_{i=0}^{m} \Gamma_{i}$ its boundary and $d(\Omega)$ its diameter. Moreover, we assume that all the edges $\Gamma_{i}$ have strictly positive measure. The system under consideration is given by

$$
\begin{cases}L u=f & \text { a.e in } \Omega, \\ \sigma(u) \cdot \overrightarrow{n_{i}}=g_{i} & \text { on }\left(\Gamma-\Gamma_{0}\right) \cap \Gamma_{i}, 1 \leq i \leq m, \\ u=0 & \text { on } \Gamma_{0} .\end{cases}
$$

*Corresponding author. Email addresses: djamel .aitakli@ummto.dz (D. Ait-akli), kader .merakeb@ummto.dz (A. Merakeb) 
We need to assume that the edges $\Gamma_{i}$ which form the boundary $\Gamma$ fulfill a condition similar to assumption $\left(\mathrm{H}_{2}\right)$ in ([2], Theorem 2.3). Actually, for our purpose, a stronger condition is needed and it is formulated in (1.5) below. The vector function $u=\left(u^{1}, u^{2}\right)$ satisfying the system (1.8) describes a displacement in the plane. In this model we impose a homogeneous Dirichlet condition on $\Gamma_{0}$ and a Neumann condition on the remaining part of the boundary. The equality on the boundary is understood in the sense of the trace. We denote $L$ the Lamé operator defined by

$$
L u:=-\operatorname{div} \sigma(u)=-\operatorname{div}[2 \mu \varepsilon(u)+\lambda \operatorname{Tr} \varepsilon(u) I d] .
$$

We assume the data functions $f$ and $g$ at the right hand sides to satisfy $f \in\left[L^{2}(\Omega)\right]^{2}$ and $g \in\left[H^{\frac{1}{2}}\left(\Gamma-\Gamma_{0}\right)\right]^{2}$. The vector $\overrightarrow{n_{i}}$ represents the outside normal to $\Gamma_{i}$. We write $\mu$ and $\lambda$ the Lamé's coefficients. We place ourselves in the isotropic framework, the deformation tensor $\varepsilon$ is defined by

$$
\varepsilon(u)=\frac{1}{2}\left(\nabla u+\nabla^{t} u\right) .
$$

The weak form of problem (1.1) is (see [1,3]): Find $u \in V$ such that $\forall v \in V$

$$
\int_{\Omega} 2 \mu \varepsilon(u) \varepsilon(v)+\lambda \operatorname{div} u \operatorname{div} v \mathrm{~d} x=\int_{\Omega} f v \mathrm{~d} x+\int_{\Gamma-\Gamma_{0}} g v \mathrm{~d} \sigma(x),
$$

where

$$
V=\left\{v \in\left[H^{1}(\Omega)\right]^{2} ; \quad v=0 \quad \text { on } \Gamma_{0}\right\} .
$$

The existence and uniqueness issue of the solution of (1.4) in $V$ is classic, (see [3]).

If we denote $\theta$ the interior angle between the edges $\Gamma_{j}$ and $\Gamma_{k}, 0 \leq j, k \leq m$ such that $\bar{\Gamma}_{j} \cap$ $\bar{\Gamma}_{k} \neq \varnothing$ and if we denote $\gamma$ the interior angle between the Neumann part of the boundary $\Gamma_{N}:=\Gamma-\Gamma_{0}$ and the Dirichlet part of the boundary $\Gamma_{D}:=\Gamma_{0}$, then we impose

$$
0<\theta<\pi, \quad 0<\gamma<\pi .
$$

The reason behind this assumption on the boundary is to get a better regularity of the solution of the weak problem (1.4). Precisely in that case we have, following ([2], Theorem 2.3) stated at the bottom of page 330, $u \in\left[H^{\frac{3}{2}+\iota}(\Omega)\right]^{2}$ for some positive $\iota>0$, which implies in particular, using the appropriate Sobolev embedding and since $\Omega$ is a locally Lipschitz domain, see part II of ([4], Theorem 4.12, page 85), that $u \in\left[C^{0, \frac{1}{2}+\iota}(\bar{\Omega})\right]^{2}$ i.e. $u$ is $\left(\frac{1}{2}+\right.$ ı)-holder continuous. One should notice that condition (1.5) are met since the domain considered in our case is convex. Let us denote

$$
\|\varepsilon(u)\|_{0, \Omega}:=\left(\int_{\Omega} \varepsilon(u) \varepsilon(u) \mathrm{d} x\right)^{\frac{1}{2}} ; \quad\|\nabla u\|_{0, \Omega}:=\left(\int_{\Omega}\left|\nabla u^{1}\right|^{2}+\left|\nabla u^{2}\right|^{2} \mathrm{~d} x\right)^{\frac{1}{2}} .
$$

By using the second Korn inequality, see [5], the trace and the Poincaré's inequalities, one easily gets from (1.4) the following estimate

$$
\|\nabla u\|_{0, \Omega} \leq \frac{1}{c_{k}} \frac{1}{2 \mu}\left(c_{p}\|f\|_{0, \Omega}+c_{p, t}\|g\|_{\frac{1}{2}, \Gamma-\Gamma_{0}}\right),
$$


where $c_{p, t}$ is a constant that depends of Poincaré constant and the constant of trace inequality. $c_{k}$ is the constant of the Korn's inequality. Note that the value of the constant $c_{k}$ and $c_{p, t}$ appearing in (1.6) are unknown and can not be explicitly lower-bounded or upper-bounded in the general case. We propose to determine explicitly these constants. The main result of this work is stated in the following theorem:

Theorem 1.1. The unique weak solution $u$ of (1.4) on the convex polygonal domain $\Omega$ admits the explicit upper bound

$$
\|\nabla u\|_{0, \Omega} \leq \frac{1}{\sqrt{\mu}}\left(1+d(\Omega)\|f\|_{0, \Omega}+8\left(|\Gamma|+\|g\|_{0, \Gamma}^{2}\right)\right),
$$

where $d(\Omega)$ represent the diameter of $\Omega$ and $|\Gamma|$ the measure of the boundary of $\Omega$.

Estimate (1.7) is similar to estimate (1.6) in the sense that the constants appearing there are the same. Before demonstrating this theorem, it is useful to go through some remarks and results. These auxiliary results are needed in order to adequately get a decomposition of the solution of the main problem, which belongs to $H^{1}(\Omega)$, into functions that are still in $H^{\frac{1}{2}}(\Gamma)$. Denote $x_{i}$, for $1 \leq i \leq m$, the vertex of the polygon that connects $\Gamma_{i-1}$ with $\Gamma_{i}$ and $x_{0}$ the one that connects $\Gamma_{m}$ to $\Gamma_{0}$. Define the auxiliary function $u_{\epsilon} \in H^{1}(\Omega)$ as the unique solution to the following Dirichlet problem

$$
\begin{cases}L u_{\epsilon}=f & \text { a.e in } \Omega, \\ u_{\epsilon}=u_{\epsilon}^{d} & \text { on } \Gamma,\end{cases}
$$

where $u_{\epsilon}^{d}$ is the trace on the boundary $\Gamma$ of the function

$$
\phi_{\epsilon}(x) u(x) .
$$

For $\epsilon \ll \frac{\left|\Gamma_{i}\right|}{2} \forall i, 0 \leq i \leq m$, the function $\phi_{\epsilon}$ is defined by

$$
\left\{\begin{array}{l}
\phi_{\epsilon}(x)=0, \quad\left\|x-x_{i}\right\| \leq \epsilon^{2}, \quad 0 \leq i \leq m ; \\
\phi_{\epsilon}(x)=\exp \left[-\frac{\epsilon^{\frac{1}{2}}\left(\epsilon-\| x-x_{i}||\right)}{\left\|x-x_{i}\right\|-\epsilon^{2}}\right], \quad \epsilon^{2}<\left\|x-x_{i}\right\|<\epsilon, \quad 0 \leq i \leq m ; \\
\phi_{\epsilon}(x)=1, \quad \epsilon \leq\left\|x-x_{i}\right\|, \quad 0 \leq i \leq m .
\end{array}\right.
$$

Let us denote

$$
D_{i, \epsilon}:=\left\{x \in \mathbb{R}^{2} \text { such that } \quad\left\|x-x_{i}\right\|<\epsilon^{2}\right\},
$$

where $\| x-x_{i}||$ stands for the Euclidean norm of the vector $x-x_{i}$. We easily see that $\phi_{\epsilon} \in$ $C^{0}(\bar{\Omega})$, consequently, there will be no jump when passing to the distributional derivative and thus $\nabla u_{\epsilon} \in L^{2}(\Omega)$, and by using the poincaré inequality, we infer that $u_{\epsilon} \in H^{1}(\Omega)$. It is shown, using Lebesgue's dominated convergence theorem for instance, that

$$
\left\|\phi_{\epsilon}-1\right\|_{0, \Gamma_{i}} \rightarrow 0
$$


i.e. we have convergence in $L^{2}$ along the edge $\Gamma_{i}$. The functions $\phi_{\epsilon}$ are identically zero on a small neighborhood of the respective vertices of the polygon.

In the sequel, we denote $u_{\epsilon}$ the vector-valued function $u_{\epsilon}=\left(u_{\epsilon}^{1}, u_{\epsilon}^{2}\right)$.

\section{Weak problem for $u_{\epsilon}$ and approximation results}

First of all, we construct the weak problem verified by the approximating function $u_{\epsilon}$. With the approximating displacement $u_{\epsilon} \in V$ is associated the approximating stress tensor

$$
\sigma_{\epsilon}:=2 \mu \varepsilon\left(u_{\epsilon}\right)+\lambda \operatorname{Tr} \varepsilon\left(u_{\epsilon}\right) I,
$$

since $L u_{\epsilon}=\operatorname{div} \sigma_{\epsilon}=f$, then $\sigma_{\epsilon} \in[H(\operatorname{div})(\Omega)]^{2 \times 2}$. For a fixed $\epsilon$, by density of the regular functions in the space $H(\operatorname{div})(\Omega)$, there exists $\sigma_{\epsilon}^{n} \in\left[C^{\infty}(\bar{\Omega})\right]^{2 \times 2}$ such that $\sigma_{\epsilon}^{n} \rightarrow \sigma_{\epsilon}$ in $[H(\operatorname{div})(\Omega)]^{2 \times 2}$. This means

$$
\left\|\sigma_{\epsilon}^{n}-\sigma_{\epsilon}\right\|_{\operatorname{div}, \Omega}:=|| \operatorname{div} \sigma_{\epsilon}^{n}-\operatorname{div} \sigma_{\epsilon}\left\|_{0, \Omega}+\right\| \sigma_{\epsilon}^{n}-\sigma_{\epsilon} \|_{0, \Omega} \rightarrow 0
$$

when $n \rightarrow \infty$. We pose $\operatorname{div} \sigma_{\epsilon}^{n}=f^{n}$, then integrating by part against a test function $v \in$ $\left[C^{\infty}(\bar{\Omega})\right]^{2} \cap V$ yields the following

$$
\int_{\Omega} \sigma_{\epsilon}^{n} \nabla v=\int_{\Omega} f^{n} v+\int_{\Gamma} \sigma_{\epsilon}^{n} \cdot \vec{n} v \mathrm{~d} \sigma(x)
$$

Passing to the limit in $n$ using (2.2), we find $\forall v \in\left[C^{\infty}(\bar{\Omega})\right]^{2} \cap V$

$$
\int_{\Omega} \sigma_{\epsilon} \nabla v=\int_{\Omega} f v+\left\langle\sigma_{\epsilon} \cdot \vec{n}, v>_{\left[H^{\frac{1}{2}}\right]^{\prime}\left(\Gamma-\Gamma_{0}\right) \times\left[H^{\frac{1}{2}}\right]\left(\Gamma-\Gamma_{0}\right)^{\prime}}\right.
$$

where $\sigma_{\epsilon} \cdot \vec{n}=: g^{\epsilon} \in\left[H^{\frac{1}{2}}\left(\Gamma-\Gamma_{0}\right)\right]^{\prime}$ is the image of the normal component $\sigma_{\epsilon}$ by the trace operator on $\Gamma$. Since, following theorem 1 established in $[8],\left[C^{\infty}(\bar{\Omega})\right]^{2} \cap V$ is a dense subset of $V \subset H^{1}(\Omega)$, then, according to the definition (1.3) and the expression (2.1), the function $u_{\epsilon}$ satisfy

$$
\begin{aligned}
& \int_{\Omega} 2 \mu \varepsilon\left(u_{\epsilon}\right) \varepsilon(v)+\int_{\Omega} \lambda \operatorname{div} u_{\epsilon} \operatorname{div} v \\
= & \int_{\Omega} f v+\left\langle g^{\epsilon}, v>_{\left[H^{\frac{1}{2}}\right]^{\prime}\left(\Gamma-\Gamma_{0}\right) \times H^{\frac{1}{2}}\left(\Gamma-\Gamma_{0}\right)^{\prime}} \quad \forall v \in V,\right.
\end{aligned}
$$

this is the weak problem satisfied by the approximating function $u_{\epsilon}$.

Let us recall, (see [6]), that the $H^{\frac{1}{2}}$-norm in one dimension on $\Gamma_{i}$ is defined by

$$
\|u\|_{\frac{1}{2}, \Gamma_{i}}:=\left(\|u\|_{0, \Gamma_{i}}^{2}+\int_{\Gamma_{i}} \int_{\Gamma_{i}} \frac{|u(x)-u(y)|^{2}}{\|x-y\|^{2}} \mathrm{~d} x \mathrm{~d} y\right)^{\frac{1}{2}} .
$$


Remark 2.1. For any sufficiently small $\epsilon>0$, it is possible to cover $\Omega$ with a collection of open sets $\left(W_{j}^{\epsilon}\right)_{j}$ such that for each $j, W_{j}^{\epsilon} \cap \Gamma$ is either empty or equals one of the following subsets: for some $i, 0 \leq i \leq m-1$

1) $\Gamma_{i}^{1, \epsilon}:=\left\{x \in \Gamma_{i} ; \quad 0<\left\|x-x_{i}\right\|<2 \epsilon\right\}$;

2) $\Gamma_{i}^{2, \epsilon}:=\left\{x \in \Gamma_{i} ; \quad 0<\left\|x-x_{i+1}\right\|<2 \epsilon\right\}$

3) $\Gamma_{i}^{3, \epsilon}:=\left\{x \in \Gamma_{i} ; \quad\left\|x-x_{i}\right\|>\frac{3}{2} \epsilon\right.$ and $\left.\quad\left\|x-x_{i+1}\right\|>\frac{3}{2} \epsilon\right\}$;

4) $\Gamma_{i}^{4, \epsilon}=\left\{x \in \Gamma_{i} \cup \Gamma_{i+1} ; \quad\left\|x-x_{i+1}\right\|<\epsilon^{2}\right\}$;

and for $i=m$

1) $\Gamma_{m}^{1, \epsilon}:=\left\{x \in \Gamma_{m} ; \quad 0<\left\|x-x_{m}\right\|<2 \epsilon\right\}$;

2) $\Gamma_{m}^{2, \epsilon}:=\left\{x \in \Gamma_{m} ; \quad 0<\left\|x-x_{0}\right\|<2 \epsilon\right\}$;

3) $\Gamma_{m}^{3, \epsilon}:=\left\{x \in \Gamma_{m} ; \quad\left\|x-x_{m}\right\|>\frac{3}{2} \epsilon \quad\right.$ and $\left.\quad\left\|x-x_{0}\right\|>\frac{3}{2} \epsilon\right\}$;

4) $\Gamma_{m}^{4, \epsilon}=\left\{x \in \Gamma_{m} \cup \Gamma_{0} ; \quad\left\|x-x_{0}\right\|<\epsilon^{2}\right\}$.

Let $\left(\vartheta_{j}^{\epsilon}\right)_{j}$, with supp $\vartheta_{j}^{\epsilon} \subset W_{j}^{\epsilon}$, a $C^{1}$-partition of unity with respect to this cover. Since $\vartheta_{j}^{\epsilon} \in H^{\frac{1}{2}}(\Gamma)$, then

$$
\begin{aligned}
& \left\|\phi_{\epsilon}-1\right\|\left\|_{\frac{1}{2}, \Gamma}=\right\|\left(\phi_{\epsilon}-1\right) \sum_{j} \vartheta_{j}^{\epsilon}\left\|_{\frac{1}{2}, \Gamma} \leq \sum_{i=0}^{m}\right\|\left(\phi_{\epsilon}-1\right) \sum_{j, \operatorname{supp} \vartheta_{j}^{\epsilon} \subset \Gamma_{i}} \vartheta_{j}^{\epsilon}\left\|_{\frac{1}{2}, \Gamma_{i}}+\sum_{i=0}^{m}\right\|\left(\phi_{\epsilon}-1\right) \|_{\frac{1}{2}, \Gamma_{i}^{4, \epsilon}} \\
\leq & \sum_{i=0}^{m}\left\|\phi_{\epsilon}-1\right\|_{\frac{1}{2}, \Gamma_{i}}+(m+1)\left\|\left(\phi_{\epsilon}-1\right)\right\|_{\frac{1}{2}, \Gamma_{0}^{4, \epsilon}}=\sum_{i=0}^{m}\left\|\phi_{\epsilon}-1\right\|_{\frac{1}{2}, \Gamma_{i}}+(m+1)\left\|\left(\phi_{\epsilon}-1\right)\right\|_{0, \Gamma_{0}^{4, \epsilon}} .
\end{aligned}
$$

One should well remark that, following its definition, the function $\phi_{\epsilon}-1$ has null $H^{\frac{1}{2}}-$ semi norm on $\Gamma_{0}^{4, \epsilon}$; this fact justify the writing of the last term in estimate (2.4). So, using the definition and symmetry of $\phi_{\epsilon}$, we get for all $0 \leq i \leq m$

$$
\begin{aligned}
& \left\|\phi_{\epsilon}-1\right\|\left\|_{\frac{1}{2}, \Gamma_{i}}=\right\|\left(\phi_{\epsilon}-1\right) \sum_{j, \text { supp } \vartheta_{j}^{\epsilon} \subset \Gamma_{i}} \vartheta_{j}^{\epsilon} \|_{\frac{1}{2}, \Gamma_{i}} \\
& \leq\left\|\left(\phi_{\epsilon}-1\right) \sum_{j, \text { supp } \vartheta_{j}^{\epsilon} \subset \Gamma_{i}^{1, \epsilon}} \vartheta_{j}^{\epsilon}\right\|_{\frac{1}{2}, \Gamma_{i}}+\left\|\left(\phi_{\epsilon}-1\right) \sum_{j, \operatorname{supp} \vartheta_{j}^{\epsilon} \subset \Gamma_{i}^{2, \epsilon}} \vartheta_{j}^{\epsilon}\right\|_{\frac{1}{2}, \Gamma_{i}} \\
& +\left\|\left(\phi_{\epsilon}-1\right) \sum_{j, \operatorname{supp} \vartheta_{j}^{\epsilon} \subset \Gamma_{i}^{3, \epsilon}} \vartheta_{j}^{\epsilon}\right\|_{\frac{1}{2}, \Gamma_{i}}+2\left\|\left(\phi_{\epsilon}-1\right)\right\| \|_{\frac{1}{2}, \Gamma_{i}^{4, \epsilon} .}
\end{aligned}
$$


Thus,

$$
\begin{aligned}
\left\|\phi_{\epsilon}-1\right\|_{\frac{1}{2}, \Gamma_{i}} & \leq \sum_{j=1}^{3}\left\|\phi_{\epsilon}-1\right\|_{\frac{1}{2}, \Gamma_{i}^{j, \epsilon}}+2\left\|\phi_{\epsilon}-1\right\|_{\frac{1}{2}, \Gamma_{i}^{4, \epsilon}} \\
& =2\left\|\phi_{\epsilon}-1\right\|_{\frac{1}{2}, \Gamma_{i}^{1, \epsilon}}+2\left\|\phi_{\epsilon}-1\right\|_{\frac{1}{2}, \Gamma_{i}^{4, \epsilon}} .
\end{aligned}
$$

Thus we have

$$
\begin{aligned}
& \left\|\phi_{\epsilon}-1\right\|_{\frac{1}{2}, \Gamma_{i}} \\
\leq & 2|| \phi_{\epsilon}-1 \mid\left\|_{0, \Gamma_{i}^{1, \epsilon}}+2\left(\int_{\Gamma_{i}^{1, \epsilon}} \int_{\Gamma_{i}^{1, \epsilon}} \frac{\left|\phi_{\epsilon}(x)-\phi_{\epsilon}(y)\right|^{2}}{\|x-y\|^{2}} \mathrm{~d} x \mathrm{~d} y\right)^{\frac{1}{2}}+2\right\|\left(\phi_{\epsilon}-1\right) \|_{0, \Gamma_{i}^{4, \epsilon}} .
\end{aligned}
$$

Lemma 2.1. The functions $\phi_{\epsilon}$ admit the following limits

$$
\text { for all } 0 \leq i \leq m \quad\left\|\phi_{\epsilon}-1\right\|_{\frac{1}{2}, \Gamma_{i}} \rightarrow 0 \quad \text { as } \quad \epsilon \rightarrow 0 .
$$

Proof. If we choose the vertex point $x_{i}$ as the origin of the $\mathbb{R}^{2}$-orthonormal coordinate system such that $\Gamma_{i}$ is supported by the positive half $x$-axis then the abscisses of $x \in$ $\left.\Gamma_{i}^{1, \epsilon} \equiv\right] 0,2 \epsilon[$ satisfy

$$
|| x-x_{i}||=|x|=x .
$$

The $H^{\frac{1}{2}}$-semi-norm of $\phi_{\epsilon}$ on $\Gamma_{i}$ writes

$$
\left|\phi_{\epsilon}-1\right|_{\frac{1}{2}, \Gamma_{i}}^{2}:=\int_{\Gamma_{i}} \int_{\Gamma_{i}} \frac{\left|\phi_{\epsilon}(x)-\phi_{\epsilon}(y)\right|^{2}}{\| x-\left.y\right|^{2}} \mathrm{~d} x \mathrm{~d} y
$$

It yields by using the definition of $\phi_{\epsilon}$

$$
\left|\phi_{\epsilon}-1\right|_{\frac{1}{2}, \Gamma_{i}}^{2} \leq 2 \int_{0}^{\epsilon} \int_{0}^{\epsilon} \frac{\left|\phi_{\epsilon}(x)-\phi_{\epsilon}(y)\right|^{2}}{|x-y|^{2}} \mathrm{~d} x \mathrm{~d} y .
$$

Consider the decomposition of (2.7) into four partial double integrals

$$
1): \quad \int_{0}^{\epsilon^{2}} \int_{0}^{\epsilon^{2}} \frac{\left|\phi_{\epsilon}(x)-\phi_{\epsilon}(y)\right|^{2}}{|x-y|^{2}} \mathrm{~d} x \mathrm{~d} y=0
$$

this is obvious.

$$
\begin{aligned}
2): & \int_{\epsilon^{2}}^{\epsilon} \int_{\epsilon^{2}}^{\epsilon} \frac{\left|\phi_{\epsilon}(x)-\phi_{\epsilon}(y)\right|^{2}}{|x-y|^{2}} \mathrm{~d} x \mathrm{~d} y \\
\leq & \int_{\epsilon^{2}}^{\epsilon} \int_{\epsilon^{2}}^{\epsilon} \frac{\left|\exp \left[-\frac{\epsilon^{\frac{1}{2}}(\epsilon-x)}{\left(x-\epsilon^{2}\right)}\right]-\exp \left[-\frac{\epsilon^{\frac{1}{2}}(\epsilon-y)}{\left(y-\epsilon^{2}\right)}\right]\right|^{2}}{|x-y|^{2}} \mathrm{~d} x \mathrm{~d} y .
\end{aligned}
$$


The function $F(x):=\exp \left[-\frac{\epsilon^{\frac{1}{2}}(\epsilon-x)}{\left(x-\epsilon^{2}\right)}\right]$ is $C^{1}(] \epsilon^{2}, \epsilon[)$ and thus lipschitz. We have, using the fact that $x \rightarrow F^{\prime}(x)$ is increasing on $\left[\epsilon^{2}, \frac{\epsilon}{2}\right]$, that

$$
\left|F^{\prime}(x)\right| \leq \frac{\epsilon^{\frac{1}{2}}(1-\epsilon)}{\epsilon\left(\frac{1}{2}-\epsilon\right)^{2}} \exp \left(\frac{-\epsilon^{\frac{1}{2}}}{\frac{1}{2}-\epsilon}\right)=: L_{1},
$$

$\forall x \in\left[\epsilon^{2}, \frac{\epsilon}{2}\right]$. On the other hand

$$
\left|F^{\prime}(x)\right| \leq \frac{\epsilon^{\frac{1}{2}}}{\epsilon(1-\epsilon)} \exp \left(\frac{2}{1-\epsilon}\right)=: L_{2}, \quad \forall x \in\left[\frac{\epsilon}{2}, \epsilon\right] .
$$

Therefore we conclude that

$$
\left|F^{\prime}(x)\right| \leq L:=\max \left(L_{1}, L_{2}\right) \leq L_{1}+L_{2}
$$

for all $x \in\left[\epsilon^{2}, \epsilon\right]$. This yields

$$
\begin{aligned}
& \left(\int_{\epsilon^{2}}^{\epsilon} \int_{\epsilon^{2}}^{\epsilon} \frac{\left|\phi_{\epsilon}(x)-\phi_{\epsilon}(y)\right|^{2}}{|x-y|^{2}} \mathrm{~d} x \mathrm{~d} y\right)^{\frac{1}{2}} \\
\leq & \left(\int_{\epsilon^{2}}^{\epsilon} \int_{\epsilon^{2}}^{\epsilon} \frac{\left|\frac{\epsilon^{\frac{1}{2}}}{\epsilon(1-\epsilon)} \exp \left(\frac{2}{1-\epsilon}\right)+\frac{\epsilon^{\frac{1}{2}}(1-\epsilon)}{\epsilon\left(\frac{1}{2}-\epsilon\right)^{2}} \exp \left(\frac{-\frac{\epsilon^{\frac{1}{2}}}{2}}{\frac{1}{2}-\epsilon}\right)\right|^{2}|x-y|^{2}}{|x-y|^{2}} \mathrm{~d} x \mathrm{~d} y\right)^{\frac{1}{2}} \\
\leq & \left(\int_{\epsilon^{2}}^{\epsilon} \int_{\epsilon^{2}}^{\epsilon}\left|\frac{\epsilon^{\frac{1}{2}}}{\epsilon(1-\epsilon)} \exp \left(\frac{2}{1-\epsilon}\right)\right|^{2} \mathrm{~d} x \mathrm{~d} y\right)^{\frac{1}{2}}+\left(\int_{\epsilon^{2}}^{\epsilon} \int_{\epsilon^{2}}^{\epsilon}\left|\frac{\epsilon^{\frac{1}{2}}(1-\epsilon)}{\epsilon\left(\frac{1}{2}-\epsilon\right)^{2}} \exp \left(\frac{-\frac{\epsilon^{\frac{1}{2}}}{2}}{\frac{1}{2}-\epsilon}\right)\right|^{2} \mathrm{~d} x \mathrm{~d} y\right)^{\frac{1}{2}} \\
\rightarrow & 0 \text { as } \epsilon \rightarrow 0 .
\end{aligned}
$$

One more integral is

$$
\text { 3): } \begin{aligned}
& \int_{0}^{\epsilon^{2}} \int_{\epsilon^{2}}^{\epsilon} \frac{\left|\phi_{\epsilon}(x)-\phi_{\epsilon}(y)\right|^{2}}{|x-y|^{2}} \mathrm{~d} x \mathrm{~d} y \\
& \leq \int_{0}^{\epsilon^{2}} \int_{\epsilon^{2}}^{\frac{\epsilon}{2}} \frac{1}{\left|x-\epsilon^{2}\right|^{2}} \exp ^{2}\left[-\frac{\epsilon^{\frac{1}{2}}(\epsilon-x)}{\left(x-\epsilon^{2}\right)}\right] \mathrm{d} x \mathrm{~d} y+\int_{0}^{\epsilon^{2}} \int_{\frac{\epsilon}{2}}^{\epsilon^{2}} \frac{1}{\left|x-\epsilon^{2}\right|^{2}} \exp ^{2}\left[-\frac{\epsilon^{\frac{1}{2}}(\epsilon-x)}{\left(x-\epsilon^{2}\right)}\right] \mathrm{d} x \mathrm{~d} y \\
& \leq \epsilon^{2} \int_{\epsilon^{2}}^{\frac{\epsilon}{2}} \frac{1}{\left|x-\epsilon^{2}\right|^{2}} \exp ^{2}\left[-\frac{\epsilon^{\frac{1}{2}+1}(1-\epsilon)}{2\left(x-\epsilon^{2}\right)}\right] \mathrm{d} x+\epsilon^{2} \int_{\frac{\epsilon}{2}}^{\epsilon} \frac{1}{\left|\frac{\epsilon}{2}-\epsilon^{2}\right|^{2}} \exp ^{2}\left[\frac{\epsilon^{\frac{1}{2}}(2 \epsilon)}{\left(\frac{\epsilon}{2}-\epsilon^{2}\right)}\right] \mathrm{d} x \\
& \left.\leq \frac{\epsilon^{\frac{1}{2}} \exp \left(-\frac{\epsilon^{\frac{3}{2}}(1-\epsilon)}{x}\right)}{1-\epsilon}\right]_{x=0}^{x=\epsilon}+\frac{1}{\left(\frac{1}{2}-\epsilon\right)^{2}} \frac{\epsilon}{2} \exp ^{2}\left(\frac{2 \epsilon^{\frac{1}{2}}}{\left(\frac{1}{2}-\epsilon\right)}\right) \\
& \rightarrow 0 \text { as } \epsilon \rightarrow 0 .
\end{aligned}
$$


Here we used the properties of the exponential function and elementary majorizations. This final integral is

$$
\text { 4): } \quad \int_{\epsilon^{2}}^{\epsilon} \int_{0}^{\epsilon^{2}} \frac{\left|\phi_{\epsilon}(x)-\phi_{\epsilon}(y)\right|^{2}}{|x-y|^{2}} \mathrm{~d} x \mathrm{~d} y=\int_{\epsilon^{2}}^{\epsilon} \int_{0}^{\epsilon^{2}} \frac{\left|\exp \left[-\frac{\epsilon^{\frac{1}{2}}(y-\epsilon)}{\left(y-\epsilon^{2}\right)}\right]\right|^{2}}{|x-y|^{2}} \mathrm{~d} x \mathrm{~d} y \rightarrow 0,
$$

proceed in the same way as for 3 ).

Combining these four integrals on one hand and using the facts:

$$
\left\|\phi_{\epsilon}-1\right\|_{0, \Gamma} \rightarrow \text { and } 2\left\|\left(\phi_{\epsilon}-1\right)\right\|_{0, \Gamma_{i}^{4, \epsilon}} \rightarrow 0
$$

for all $0 \leq i \leq m$ on the other hand yield, using (2.5), the result of Lemma 2.1. Consequently, using (2.4) yields also

$$
\left\|\phi_{\epsilon}-1\right\|_{\frac{1}{2}, \Gamma} \rightarrow 0
$$

This completes the proof of the lemma.

Since $u$ is $\left(\frac{1}{2}+\iota\right)-$ Hölder continuous and thus uniformly continuous on $\Omega$, the result of Lemma 2.1 implies

$$
\left\|u_{\epsilon}-u\right\|_{\frac{1}{2}, \Gamma} \leq\left\|u\left(\phi_{\epsilon}-1\right)\right\|_{\frac{1}{2}, \Gamma} \leq\|u\|_{\infty, \Gamma}|| \phi_{\epsilon}-1 \|_{\frac{1}{2}, \Gamma} \rightarrow 0 .
$$

One can now prove the following approximation lemma:

Lemma 2.2. The function $u_{\epsilon}$ defined by (1.8) and the distribution $g^{\epsilon}$ appearing in problem (2.3) satisfy respectively the following limits

$$
\text { a) }\left\|\nabla u_{\epsilon}-\nabla u\right\|_{0, \Omega} \rightarrow 0, \quad \text { b) }\left\|g^{\epsilon}-g\right\|_{\left[H^{\left.\frac{1}{2}\right]^{\prime}, \Gamma-\Gamma_{0}}\right.} \rightarrow 0
$$

as $\epsilon \rightarrow 0$.

Proof. a) let us consider the following problem

$$
\begin{cases}L u=0 & \text { a.e in } \Omega, \\ u=u^{d} & \text { on } \Gamma .\end{cases}
$$

Consider the linear operator $G$ that associates to each $u^{d} \in H^{\frac{1}{2}}(\Gamma)$ the corresponding $u$ nique solution $u$ of problem (2.8),

$$
G:\left(H^{\frac{1}{2}}(\Gamma),\|\cdot\|_{\frac{1}{2}, \Gamma}\right) \rightarrow A \subset V, \quad u^{d}:=u_{\mid \Gamma} \rightarrow K\left(u^{d}\right)=u,
$$

where $\left(A,\|\cdot\|_{H^{1}(\Omega)}\right)$ denote the range of $H^{\frac{1}{2}}(\Gamma)$ under $G$. The inverse operator $G^{-1}$ identifies with the trace operator applied to $u \in A$ on $\Gamma$. This operator is obviously well defined and bijective. Using the trace inequality on $\Gamma$, there exists $c>0$ such that $\forall u \in A$

$$
\left\|G^{-1} u\right\|_{\frac{1}{2}, \Gamma}:=\|u\|_{\frac{1}{2}, \Gamma} \leq \mathcal{C}_{-1}\|\nabla u\|_{0, \Omega}
$$


this implies the continuity of the linear bijective operator $G^{-1}$. According to the Banach isomorphism theorem, the operator $G$ is continuous, this means that there exists $c>0$ such that for all $u \in A$ we have

$$
\|\nabla u\|_{0, \Omega} \leq c|| u \|_{\frac{1}{2}, \Gamma}
$$

Thus, since $L\left(u-u_{\epsilon}\right)=0$, using the limit established in Lemma 2.1

$$
\left\|\nabla u-\nabla u_{\epsilon}\right\|_{0, \Omega} \leq c|| u-u_{\epsilon} \|_{\frac{1}{2}, \Gamma} \rightarrow 0,
$$

this proves $a$ ).

b) We make the same reasoning as for $a)$. Given $g \in\left[H^{\frac{1}{2}}\left(\Gamma-\Gamma_{0}\right)\right]^{\prime}$, let $w \in V$ be the unique solution of

$$
\int_{\Omega} 2 \mu \varepsilon(w) \varepsilon(v)+\lambda \operatorname{div} w \operatorname{div} v \mathrm{~d} x=\left\langle g, v>_{\left[H^{\frac{1}{2}}\left(\Gamma-\Gamma_{0}\right)\right]^{\prime}, H^{\frac{1}{2}}\left(\Gamma-\Gamma_{0}\right)^{\prime}}\right.
$$

for all $v \in V$. Choosing $v=w$, there exists $c^{\prime}>0$ such that

$$
\|\nabla w\|_{0, \Omega} \leq c^{\prime}\|g\|_{\left[H^{\frac{1}{2}}\left(\Gamma-\Gamma_{0}\right)\right]^{\prime}}
$$

Let $K$ be the operator that associates to each data $g \in\left[H^{\frac{1}{2}}\left(\Gamma-\Gamma_{0}\right)\right]^{\prime}$ the solution function $w$ of the corresponding problem (2.10):

$$
\begin{aligned}
K:\left[H^{\frac{1}{2}}\left(\Gamma-\Gamma_{0}\right)\right]^{\prime} & \rightarrow D \subset V \\
g & \rightarrow K(g)=w,
\end{aligned}
$$

where $\left(D,\|\|_{H^{1}(\Omega)}\right)$ denote the range of $\left[H^{\frac{1}{2}}\right]^{\prime}\left(\Gamma-\Gamma_{0}\right)$ under $K$. Following existence and uniqueness result for problem (2.10), $K$ is well defined, furthermore it is linear and invertible. An equivalent formulation of (2.11) is: there exists a constant $c^{\prime}>0$ such that $\forall g \in\left[H^{\frac{1}{2}}\left(\Gamma-\Gamma_{0}\right)\right]^{\prime}$, we have

$$
\|K(g)\|_{H^{1}} \leq c^{\prime}\|g\|_{\left[H^{\frac{1}{2}}\right]^{\prime}\left(\Gamma-\Gamma_{0}\right)}
$$

i.e. $K$ is continuous. Then, according to Banach's isomorphism theorem, we deduce that $\exists c_{-1}^{\prime}>0$ such that

$$
\|g\|_{\left[H^{\frac{1}{2}}\left(\Gamma-\Gamma_{0}\right)\right]^{\prime}} \leq c_{-1}^{\prime}\|\nabla w\|_{0, \Omega} .
$$

Rewriting (1.4) with $g \in H^{\frac{1}{2}}\left(\Gamma-\Gamma_{0}\right) \equiv D \subset\left[H^{\frac{1}{2}}\left(\Gamma-\Gamma_{0}\right)\right]^{\prime}$ then subtracting (1.4) and (2.3) member-to-member, one find that $u-u_{\epsilon}$ satisfy: $\forall v \in V$,

$$
\int_{\Omega} 2 \mu\left(\varepsilon\left(u_{\epsilon}\right)-\varepsilon(u)\right) \varepsilon(v)+\lambda \operatorname{div}\left(u_{\epsilon}-u\right) \operatorname{div} v \mathrm{~d} x
$$




$$
=\left\langle g^{\epsilon}-g, v>{ }_{\left[H^{\frac{1}{2}}\left(\Gamma-\Gamma_{0}\right)\right]^{\prime}, H^{\frac{1}{2}}\left(\Gamma-\Gamma_{0}\right)} .\right.
$$

Applying (2.12) to $w=u_{\epsilon}-u$ we get:

$$
\left\|g^{\epsilon}-g\right\|_{\left[H^{\frac{1}{2}}\left(\Gamma-\Gamma_{0}\right)\right]^{\prime}} \leq c_{-1}^{\prime}\left\|\nabla u_{\epsilon}-\nabla u\right\|_{0, \Omega} .
$$

Considering (2.9), we infer $b$ ).

Remark 2.2. A consequence of the previous lemma: for arbitrary small $\beta>0$, there exists $\epsilon_{1}>0$ such that $\forall \epsilon, 0<\epsilon<\epsilon_{1}$, we have

i) $\left\|g^{\epsilon}-g\right\|_{\left[H^{\frac{1}{2}}\left(\Gamma-\Gamma_{0}\right)\right]^{\prime}} \leq \beta$,

ii) $\left\|\nabla u_{\epsilon}-\nabla u\right\|_{0, \Omega} \leq \beta$.

iii) Since, by assumption, $g \in H^{\frac{1}{2}}\left(\Gamma-\Gamma_{0}\right)$ then, using the continuity of the canonical embedding

$$
I: H^{\frac{1}{2}}\left(\Gamma-\Gamma_{0}\right) \rightarrow L^{2}\left(\Gamma-\Gamma_{0}\right),
$$

we have $\|g\|_{0, \Gamma-\Gamma_{0}} \leq\|g\|_{\frac{1}{2}, \Gamma-\Gamma_{0}}$.

\section{Auxiliary lemmas}

Let $\epsilon_{1}$ be such as defined in remark (2.2). For the rest of the paper, we fix $\epsilon, 0<\epsilon<\epsilon_{1}$.

Before presenting a proof of the main result, we intend to state two auxiliary lemmas. These lemmas are established using, principally, ([7], Theorem 2.1). In order to apply this later result, assumption 1.2 in that same paper need to be met. Our polygonal domain satisfy very well that assumption.

These lemmas, Lemmas 3.1 and 3.2 below, convey the essential idea in the demonstration of the main theorem 1.1. We begin with approximating the solution $u_{\epsilon}$ of problem (1.8) by a smooth function vanishing on an adequate part of the boundary $\Gamma$. Then the idea is to carry out a particular decomposition, alluded to in the introduction, of this approximating function. This key idea is illustrated in the following example. Consider $v \in V$ to be the solution of a problem similar to (1.8), e.g.,

$$
\begin{cases}L v=f & \text { a.e in } \Omega, \\ v=v^{d} & \text { on } \cup_{j} \Gamma_{j} .\end{cases}
$$

We assume that the trace of $v$ on the boundary vanish on an open neighborhood of the vertices of $\Omega$. Set

$$
E_{i}=\left\{v \in V ; \quad v=0 \quad \text { on } \Gamma_{i}\right\} .
$$


Let us decompose this problem into two other problems

$$
S_{1}^{i}\left\{\begin{array} { l l } 
{ L v _ { 1 } ^ { i } = f _ { 1 } } & { \text { a.e in } \Omega , } \\
{ v _ { 1 } ^ { i } = v ^ { d } } & { \text { on } \Gamma _ { i } , } \\
{ v _ { 1 } ^ { i } = 0 } & { \text { on } \cup _ { j } \Gamma _ { j } - \Gamma _ { i } , }
\end{array} \quad S _ { 2 } ^ { i } \left\{\begin{array}{ll}
L v_{2}^{i}=f_{2} & \text { a.e in } \Omega, \\
v_{2}^{i}=0 & \text { on } \Gamma_{i}, \\
v_{2}^{i}=v^{d} & \text { on } \cup_{j} \Gamma_{j}-\Gamma_{i} .
\end{array}\right.\right.
$$

Provided that the problems are well posed, the functions $v_{1}^{i}$ and $v_{2}^{i}$ are well defined. On the other hand, it is easy to see that the fact $v_{1}^{i}=v$ on $\Gamma_{i}$ does not generally imply that $\sigma\left(v_{1}^{i}\right) \cdot \vec{n}=\sigma(v) \cdot \vec{n}$ on $\Gamma_{i}$. So the idea is to find a decomposition that makes such a property to be fulfilled. i.e. such that $v_{1}^{i}$ can be approximated by a function $v_{1}^{i, n}$ and, at the same time, such that $\sigma\left(v_{1}^{i, n}\right) \cdot \vec{n}$ is an approximation of $\sigma\left(v_{1}^{i}\right) \cdot \vec{n}$ on $\Gamma_{i}$ in the sense of trace. An adequate use of Theorem 2.1 proved in [7] can make this decomposition possible.

Using ([8], Theorem 1), we can find $\left(u_{\epsilon}^{p}\right)_{p} \in C^{\infty}(\bar{\Omega})$ such that for each $p, u_{\epsilon}^{p}$ vanishes on the same part of $\Gamma$ where does $u_{\epsilon}$ and such that

$$
\left\|\nabla u_{\epsilon}^{p}-\nabla u_{\epsilon}\right\|_{0, \Omega} \rightarrow 0 \quad \text { as } p \rightarrow \infty .
$$

If we denote

$$
f^{p}:=L\left(u_{\epsilon}^{p}\right) \quad g_{p}^{\epsilon}:=\sigma\left(u_{\epsilon}^{p}\right) \cdot \vec{n}
$$

then, a particular consequence of (3.3) is: we can fix $p$ such that

$$
\left\|\nabla u_{\epsilon}^{p}-\nabla u_{\epsilon}\right\|_{0, \Omega} \leq \frac{1}{4 C_{6} \sqrt{\mu}}
$$

where $C_{6}$ is the continuity constant of the operator $G^{\prime-1}$ defined as follows. Let $G^{\prime}$ be the operator that associates to each data $(f, g) \in L^{2}(\Omega) \times\left[H^{\frac{1}{2}}\left(\Gamma-\Gamma_{0}\right)\right]^{\prime}$ the solution $v$ of

$$
\begin{cases}L v=f & \text { a.e in } \Omega, \\ \sigma(v) \cdot \vec{n}=g & \text { on } \Gamma-\Gamma_{0}, \\ v=0 & \text { on } \Gamma_{0},\end{cases}
$$

i.e.

$$
\begin{aligned}
G^{\prime}: L^{2}(\Omega) \times\left[H^{\frac{1}{2}}\left(\Gamma-\Gamma_{0}\right)\right]^{\prime} & \rightarrow M \subset V \\
(f, g) & \rightarrow G^{\prime}(f, g)=v,
\end{aligned}
$$

where $\left(M,\|\|_{H^{1}(\Omega)}\right)$ denote the range of $L^{2}(\Omega) \times\left[H^{\frac{1}{2}}\left(\Gamma-\Gamma_{0}\right)\right]^{\prime}$ under $G^{\prime}$. Since $G^{\prime}$ is bijective and continuous then, applying the Banach theorem with $G^{\prime}$, we infer that the inverse operator $G^{\prime-1}$ is continuous i.e. $\forall v \in M$

$$
\left\|G^{\prime-1} v\right\|_{L^{2}(\Omega) \times H^{\frac{1}{2}}\left(\Gamma-\Gamma_{0}\right)} \leq C_{6}\|v\|_{M} .
$$


Thus by using (3.5) we obtain, with $v=u_{\epsilon}^{p}-u_{\epsilon}$, and given an arbitrary small $\beta>0$

$$
\begin{aligned}
& \text { (i) }\left\|f^{p}-f\right\|_{0, \Omega} \leq C_{6}|| \nabla u_{\epsilon}^{p}-\nabla u_{\epsilon} \|_{0, \Omega} \leq \frac{1}{4 \sqrt{\mu}}, \\
& \text { (ii) }\left\|g_{p}^{\epsilon}-g^{\epsilon}\right\|_{\left[H^{\frac{1}{2}}\left(\Gamma-\Gamma_{0}\right)\right]^{\prime}} \leq \beta .
\end{aligned}
$$

We fix, for the rest of the paper, $p$ for which (3.5)-(3.7) are fulfilled.

Lemma 3.1. Let $\delta_{0}$ be an arbitrary small number. There exists two functions $u_{1}$ and $u_{2}$ such that

i) $u_{\epsilon}^{p}=u_{1}+u_{2}$.

ii) $\left\{\begin{array}{ll}L\left(u_{1}\right)=f_{1} & \text { in } \Omega, \\ u_{1}=0 & \text { on } \Gamma-\Gamma_{0}, \\ u_{1}=0 & \text { on } \Gamma_{0} .\end{array} \quad \begin{cases}L\left(u_{2}\right)=f_{2} & \text { in } \Omega, \\ u_{2}=u_{\epsilon}^{p} & \text { on } \Gamma-\Gamma_{0}, \\ u_{2}=0 & \text { on } \Gamma_{0} .\end{cases}\right.$

iii) $\sigma\left(u_{1}\right) \cdot \vec{n}=0$ on $\Gamma$ and thus, $\sigma\left(u_{2}\right) \cdot \vec{n}=g_{p}^{\epsilon}$ on $\Gamma$.

iv) $f_{2}:=f^{p}-f_{1}$ satisfy $\left\|f^{p}-f_{1}\right\|_{0, \Omega} \leq \frac{\delta_{0}}{2 m}$.

Proof. Let $\delta_{0}>0$ be an arbitrary small number. Consider the decomposition

$$
u_{\epsilon}^{p}:=w+z
$$

where $w$ and $z$ are respectively the solutions of the following problems

$$
\left\{\begin{array} { l l } 
{ L ( w ) = f ^ { p } } & { \text { in } \Omega , } \\
{ w = 0 } & { \text { on } \Gamma - \Gamma _ { 0 } , } \\
{ w = 0 } & { \text { on } \Gamma _ { 0 } . }
\end{array} \quad \left\{\begin{array}{ll}
L(z)=0 & \text { in } \Omega, \\
z=u_{\epsilon}^{p} & \text { on } \Gamma-\Gamma_{0}, \\
z=0 & \text { on } \Gamma_{0} .
\end{array}\right.\right.
$$

Observe that $\sigma(w) \cdot \vec{n}$ does not necessary equal zero and thus $\sigma(z) \cdot \vec{n}$ does not necessary equal $g$. Nevertheless, by density, there is functions $w_{n} \in C_{0}^{\infty}(\Omega)$ such that

$$
\left\|\nabla w_{n}-\nabla w\right\|_{0, \Omega} \rightarrow 0 \quad \text { as } n \rightarrow \infty
$$

One should note that, following the definition of compactly supported functions in $\Omega, \sigma\left(w_{n}\right) \cdot \vec{n}=0$ on $\partial \Omega$ for all $n$.

Using the same argument as in the proof of Lemma 2.2 (Banach theorem), and for the fixed homogeneous Dirichlet boundary condition, there exists $C_{0}>0$ depending merely on the domain $\Omega$ such that

$$
\left\|L\left(w_{n}\right)-f^{p}\right\|_{0, \Omega} \leq C_{0}\left\|\nabla w_{n}-\nabla w\right\|_{0, \Omega} \rightarrow 0 \quad \text { as } n \rightarrow \infty .
$$


Thus, there exists an element $w^{*}$ in $\left\{w_{n}\right\}_{n \in \mathbb{N}}$ that satisfy the estimate

$$
\left\|L\left(w^{*}\right)-f^{p}\right\|_{0, \Omega} \leq \frac{\delta_{0}}{m} .
$$

Decompose $w:=w^{*}+w_{2}$ where $w_{2}$ is a solutions of

$$
\begin{cases}L\left(w_{2}\right)=f^{p}-L\left(w^{*}\right) & \text { in } \Omega, \\ w_{2}=0 & \text { on } \Gamma-\Gamma_{0}, \\ w_{2}=0 & \text { on } \Gamma .\end{cases}
$$

One should remark that

$$
\sigma\left(w_{2}\right) \cdot \vec{n}+\sigma\left(z_{1}\right) \cdot \vec{n}=g_{p}^{\epsilon} .
$$

Indeed, $\sigma\left(w_{2}\right) \cdot \vec{n}+\sigma\left(z_{1}\right) \cdot \vec{n}=\sigma(u) \cdot \vec{n}-\sigma\left(w^{*}\right) \cdot \vec{n}=g_{p}^{e}-0$. Pose

$$
\begin{aligned}
& u_{1}:=w^{*}, \quad u_{2}:=w_{2}+z_{1}, \\
& f_{1}:=L\left(w^{*}\right), \quad f_{2}:=f^{p}-L\left(w^{*}\right),
\end{aligned}
$$

this concludes the lemma.

It should be pointed out that we repeatedly apply the essential argument in Lemma 2.2, namely the Banach isomorphism theorem, in order to obtain estimates for the inverse of some operators. On the other hand, although the functions $u_{1}$ and $u_{2}$ depends on $\epsilon$ we have removed the index $\epsilon$ for clarity of presentation.

Lemma 3.2. Let $E_{i}$ be the subspace of $V$ defined by (3.2). Let $u_{1}, u_{2}$ be such as defined in Lemma 3.1. Given $\delta_{1}, \delta_{2}>0$ arbitrary small numbers, there exists $m$ functions $u_{2, i} \in E_{i}, 1 \leq i \leq m$, such that

$$
u_{2, i}=0 \quad \text { on } \Gamma-\Gamma_{i}
$$

and such that $g_{i}:=\sigma\left(u_{2, i}\right) \cdot \vec{n}$ satisfy

$$
\left\|g_{i}-g_{p}^{\epsilon}\right\|_{0, \Gamma_{i}} \leq \frac{\delta_{1}}{m}
$$

Moreover, $u_{2}^{\prime}:=\sum_{i=1}^{m} u_{2, i}$ satisfy

$$
\left\|\nabla\left(u_{1}+u_{2}^{\prime}\right)-\nabla u_{\epsilon}^{p}\right\|_{0, \Omega} \leq \delta_{2} .
$$

The construction of the function $u_{2, i}$ that is presented in the proof of Lemma 3.2 below shows that for all $i$, the functions $u_{2, i}$ vanish not only on $\Gamma$ but also on $N_{i} \cap \partial \Omega$, where $N_{i} \subset \mathbb{R}^{2}$ is an open neighborhood of $\Gamma_{i}$. The proof is done in two main steps. In the first one, we construct the functions $u_{2, i}$ and in the second step we prove that these functions actually satisfy estimates (3.10)-(3.11). 
Proof. Let $\delta_{1}, \delta_{2}$ be arbitrary small numbers. The functions $u_{2, i}$ will be defined by introducing the intermediate functions $X_{i}, Y_{i}, \tilde{Y}_{i}^{n}$ and $V_{i}$ respectively in subsections $\left.\left.a\right), b\right), c$ ) and $d)$. As we define these functions, we demonstrate some estimates that will be used to derive (3.10)-(3.11) in the second step.

Step 1: Fix $i, 1 \leq i \leq m$. Consider the decomposition

$$
u_{2}=X_{i}+\sum_{j \neq i} X_{j}=X_{i}+Z_{i}
$$

where $X_{i} \in E_{i}$ and $Z_{i}$ are the solutions of

$$
\left(P_{1}\right)\left\{\begin{array} { l l } 
{ L ( X _ { i } ) = 0 } & { \text { in } \Omega , } \\
{ X _ { i } = u _ { \epsilon } ^ { p } } & { \text { on } \Gamma _ { i } , } \\
{ X _ { i } = 0 } & { \text { on } \Gamma - \Gamma _ { i } \cup \Gamma _ { 0 } , } \\
{ X _ { i } = 0 } & { \text { on } \Gamma _ { 0 } . }
\end{array} \quad ( P _ { 1 } ^ { \prime } ) \left\{\begin{array}{ll}
L\left(Z_{i}\right)=f_{2} & \text { in } \Omega, \\
Z_{i}=0 & \text { on } \Gamma_{i}, \\
Z_{i}=u_{\epsilon}^{p} & \text { on } \Gamma-\Gamma_{i} \cup \Gamma_{0}, \\
Z_{i}=0 & \text { on } \Gamma_{0} .
\end{array}\right.\right.
$$

One should notice that these two problems are well posed, this can be seen by considering the regularity of $u_{\epsilon}^{p}$ on the boundary and the fact that $u_{\epsilon}^{p}$ vanish identically on a neighborhood of the the vertices. Since $\Omega$ is a Lipschitz domain, it posses the $W^{1,2}$ Sobolev extension property.

a) According to ([7, Theorem 2.1]) see Appendix $\mathrm{A}$ at the end, there exists $X_{i}^{n} \in$ $C_{0}^{\infty}\left(\mathbb{R}^{2}\right)$ such that we have

$$
\text { (h1) }\left\|\nabla X_{i}^{n}-\nabla X_{i}\right\|_{0, \Omega} \leq \frac{\delta_{3}}{m}, \quad(h 2) \operatorname{supp}\left(X_{i}^{n}\right) \cap\left(\Gamma-\Gamma_{i}\right)=\varnothing,
$$

where $\delta_{3}>0$ is an adequately chosen real number. A consequence of $(h 2)$ is:

$$
\text { (h3) } \sigma\left(X_{i}^{n}\right) \cdot \vec{n}=0 \quad \text { on } \Gamma-\Gamma_{i}, \quad X_{i}^{n}=0 \quad \text { on } \Gamma-\Gamma_{i} .
$$

Furthermore, with the homogeneous Dirichlet condition on $\Gamma-\Gamma_{i}$ being fixed in the problem $P_{1}$ and in the problem solved by $X_{i}^{n}$ then, using the Banach theorem, there exists $C_{1}>0$ such that

$$
\begin{aligned}
& \left\|\sigma\left(X_{i}^{n}\right) \cdot \vec{n}-\sigma\left(X_{i}\right) \cdot \vec{n}\right\|_{0, \Gamma_{i}}+\left\|L\left(X_{i}^{n}\right)-0\right\|_{0, \Omega} \\
\leq & C_{1}\left\|\nabla X_{i}^{n}-\nabla X_{i}\right\|_{0, \Omega} \leq \frac{C_{1} \delta_{3}}{m} .
\end{aligned}
$$

We choose $\delta_{3}$ in assumption $(h 1)$ such that

$$
\text { (c1): } \frac{C_{1} \delta_{3}}{m}<\frac{\delta_{0}}{4 m}
$$

where $\delta_{0}$ is a positive real number whose choice will be precised later (see condition $(c 4)$ below). Fix $n_{1}$ such that $F_{i}^{n_{1}}:=L\left(X_{i}^{n_{1}}\right)$ satisfy

$$
\left\|F_{i}^{n_{1}}-0\right\|_{0, \Omega} \leq \frac{\delta_{0}}{4 m}
$$


Denote $\tilde{X}_{i}^{n_{1}}:=X_{i}-X_{i}^{n_{1}} \in E_{i}$, it does satisfy

$$
X_{i}^{n_{1}}+\tilde{X}_{i}^{n_{1}}+Z_{i}=u_{\epsilon}^{p}
$$

or equivalently,

$$
\left(P_{2}\right)\left\{\begin{array} { l l } 
{ L ( X _ { i } ^ { n _ { 1 } } ) = F _ { i } ^ { n } } & { \text { in } \Omega , } \\
{ X _ { i } ^ { n _ { 1 } } = x _ { i } ^ { n _ { 1 } } } & { \text { on } \Gamma _ { i } , } \\
{ X _ { i } ^ { n _ { 1 } } = 0 } & { \text { on } \Gamma - \Gamma _ { i } . }
\end{array} \quad ( P _ { 2 } ^ { \prime } ) \left\{\begin{array}{ll}
L\left(\tilde{X}_{i}^{n_{1}}\right)=-F_{i}^{n} & \text { in } \Omega, \\
\tilde{X}_{i}^{n_{1}}=u_{\epsilon}^{p}-x_{i}^{n_{1}} & \text { on } \Gamma_{i}, \\
\tilde{X}_{i}^{n_{1}}=0 & \text { on } \Gamma-\Gamma_{i},
\end{array}\right.\right.
$$

for the same reasons as for $\left(P_{1}\right)$ and $\left(P_{1}^{\prime}\right)$, problems $\left(P_{2}\right)$ and $\left(P_{2}^{\prime}\right)$ are well posed. Considering (h3) and (3.13), the function $\tilde{X}_{i}^{n_{1}}+Z_{i}$ satisfy

$$
\left(P_{3}\right) \begin{cases}L\left(\tilde{X}_{i}^{n_{1}}+Z_{i}\right)=-F_{i}^{n_{1}}+f_{2} & \text { in } \Omega, \\ \tilde{X}_{i}^{n_{1}}+Z_{i}=u_{\epsilon}^{p}-x_{i}^{n_{1}} & \text { on } \Gamma_{i}, \\ \sigma\left(\tilde{X}_{i}^{n_{1}}+Z_{i}\right) \cdot \vec{n}=g_{p}^{\epsilon} & \text { on } \Gamma-\Gamma_{i} \cup \Gamma_{0}, \\ \tilde{X}_{i}^{n_{1}}+Z_{i}=0 & \text { on } \Gamma_{0} .\end{cases}
$$

b) Let $Y_{i}$ be the function defined by

$$
\left(P_{4}\right) \begin{cases}L\left(Y_{i}\right)=-F_{i}^{n_{1}}+f_{2} & \text { in } \Omega, \\ Y_{i}=0 & \text { on } \Gamma_{i}, \\ \sigma\left(Y_{i}\right) \cdot \vec{n}=g_{p}^{e} & \text { on } \Gamma-\Gamma_{i} \cup \Gamma_{0}, \\ Y_{i}=0 & \text { on } \Gamma_{0},\end{cases}
$$

thanks to assumption (1.5), this problem is well posed. There exists a trace constant $c_{t}$ such that, using $(h 1)$, we have

$$
\left\|\left(u_{\epsilon}^{p}-x_{i}^{n_{1}}\right)\right\|_{0, \Gamma_{i}} \leq c_{t} \times\left\|\nabla X_{i}-\nabla X_{i}^{n_{1}}\right\|_{0, \Omega} \leq \frac{c_{t} \times \delta_{3}}{m} .
$$

For the fixed $-F_{i}^{n_{1}}+f_{2} \in L^{2}(\Omega), g_{p}^{e} \in L^{2}\left(\Gamma-\Gamma_{i} \cup \Gamma_{0}\right)$ and the homogeneous Dirichlet condition on $\Gamma_{0}$ in problems $\left(P_{3}\right)$ and $\left(P_{4}\right)$, and by using an argument similar to that in part (a) of the proof of lemma (2.2) (Banach theorem), there exists a constant $C_{3}>0$ such that

$$
\begin{aligned}
& \left\|\nabla Y_{i}-\nabla\left(\tilde{X}_{i}^{n_{1}}+Z_{i}\right)\right\|_{0, \Omega} \\
\leq & C_{3}\left\|Y_{i}-\left(u_{\epsilon}^{p}-x_{i}^{n_{1}}\right)\right\|_{0, \Gamma_{i}}=C_{3}\left\|0-\left(u_{\epsilon}^{p}-x_{i}^{n_{1}}\right)\right\|_{0, \Gamma_{i}} \leq \frac{c_{t} \times C_{3} \delta_{3}}{m},
\end{aligned}
$$

and by using the trace inequality again we have

$$
\left\|Y_{i}-\left(\tilde{X}_{i}^{n_{1}}+Z_{i}\right)\right\|_{0, \Gamma-\Gamma_{i}} \leq c_{t}\left\|\nabla Y_{i}-\nabla\left(\tilde{X}_{i}^{n_{1}}+Z_{i}\right)\right\|_{0, \Omega} \leq c_{t}^{2} \times C_{3} \frac{\delta_{3}}{m} .
$$


Considering (3.13) and (h3), we easily see that $\tilde{X}_{i}^{n_{1}}+Z_{i}=u_{\epsilon}^{p}$ on $\Gamma-\Gamma_{i}$; and thus, estimates (3.15) and (3.16) become respectively

$$
\begin{aligned}
& \left\|\nabla\left(X_{i}^{n_{1}}+Y_{i}\right)-\nabla u_{\epsilon}^{p}\right\|_{0, \Omega} \leq \frac{c_{t} \times C_{3} \delta_{3}}{m}, \\
& \left\|Y_{i}-u_{\epsilon}^{p}\right\|_{0, \Gamma-\Gamma_{i}} \leq \frac{c_{t}^{2} \times C_{3} \delta_{3}}{m} .
\end{aligned}
$$

On the other hand, since $Y_{i}=0$ on $\Gamma_{i}$ then, using the result (Theorem 2.1, [7] see Appendix A at the end), there exists $Y_{i}^{n} \in C_{0}^{\infty}\left(\mathbb{R}^{2}\right)$ such that

$$
\text { (h4) }\left\|\nabla Y_{i}^{n}-\nabla Y_{i}\right\|_{0, \Omega} \leq \frac{\delta_{1}^{\prime}}{m} \text { and } \operatorname{supp}\left(Y_{i}^{n}\right) \cap \Gamma_{i}=\varnothing,
$$

where $\delta_{1}^{\prime}>0$ is an adequately chosen real number, thus

$$
\sigma\left(Y_{i}^{n}\right) \cdot \vec{n}=0 \text { on } \Gamma_{i} \text { and } Y_{i}^{n}=0 \text { on } \Gamma_{i}
$$

for the homogeneous Dirichlet condition on $\Gamma_{i} \cup \Gamma_{0}$ for problem $\left(P_{4}\right)$ and the problem solved by $Y_{i}^{n}$, and using Banach theorem, there exists $C_{1}^{\prime}>0$ such that

$$
\left\|L\left(Y_{i}^{n}\right)-\left(-F_{i}^{n_{1}}+f_{2}\right)\right\|_{0, \Omega} \leq C_{1}^{\prime}\left\|\nabla Y_{i}^{n}-\nabla Y_{i}\right\|_{0, \Omega}
$$

We choose $\delta_{1}^{\prime}>0$ in assumption $(h 4)$ such that

$$
(c 2): \quad \frac{C_{1}^{\prime} \delta_{1}^{\prime}}{m}<\frac{\delta_{0}}{4 m}
$$

where $\delta_{0}$ is as defined in Lemma 3.1 i.e. need to satisfy condition (c4) below. Fix $n_{2}>n_{1}$ such that

$$
h_{i}^{n_{2}}:=L\left(Y_{i}^{n_{2}}\right)
$$

satisfy

$$
\left\|h_{i}^{n_{2}}-\left(-F_{i}^{n_{1}}+f_{2}\right)\right\|_{0, \Omega} \leq \frac{\delta_{0}}{4 m} .
$$

Consequently, considering (iv) of Lemmas 3.1 and 3.2 and (3.12), we have

$$
\left\|h_{i}^{n_{2}}-0\right\| \leq \frac{\delta_{0}}{m}
$$

c) Denote $\tilde{Y}_{i}^{n_{2}}:=Y_{i}-Y_{i}^{n_{2}}$, we have

$$
\left(P_{5}\right) \begin{cases}L\left(Y_{i}^{n_{2}}\right)=h_{i}^{n_{2}} & \text { in } \Omega, \\ Y_{i}^{n_{2}}=0 & \text { on } \Gamma_{i}, \\ Y_{i}^{n_{2}}=y_{i}^{n_{2}} & \text { on } \Gamma-\Gamma_{i} \cup \Gamma_{0}, \\ Y_{i}^{n_{2}}=0 & \text { on } \Gamma_{0},\end{cases}
$$




$$
\left(P_{6}\right) \begin{cases}L\left(\tilde{Y}_{i}^{n_{2}}\right)=-h_{i}^{n_{2}}-F_{i}^{n_{1}}+f_{2} & \text { in } \Omega, \\ \tilde{Y}_{i}^{n_{2}}=0 & \text { on } \Gamma_{i}, \\ \tilde{Y}_{i}^{n_{2}}=Y_{i}-y_{i}^{n_{2}} & \text { on } \Gamma-\Gamma_{i} \cup \Gamma_{0}, \\ \tilde{Y}_{i}^{n_{2}}=0 & \text { on } \Gamma_{0} .\end{cases}
$$

Rewriting estimate (3.17) gives

$$
\left\|\nabla\left(\tilde{Y}_{i}^{n_{2}}+Y_{i}^{n_{2}}+X_{i}^{n_{1}}\right)-\nabla u_{\epsilon}^{p}\right\|_{0, \Omega} \leq \frac{c_{t} \times C_{3} \delta_{3}}{m},
$$

and denote $g^{\prime}:=\sigma\left(\tilde{Y}_{i}^{n_{2}}\right) \cdot \vec{n} \in L^{2}\left(\Gamma-\Gamma_{0}\right)$.

d) Let $V_{i} \in E_{i}$ be the function defined by

$$
\left(P_{7}\right) \begin{cases}L\left(V_{i}\right)=-h_{i}^{n_{2}}-F_{i}^{n_{1}}+f_{2} & \text { in } \Omega, \\ \sigma\left(V_{i}\right) \cdot \vec{n}=g^{\prime} & \text { on } \Gamma_{i}, \\ V_{i}=0 & \text { on } \Gamma-\Gamma_{i} \cup \Gamma_{0}, \\ V_{i}=0 & \text { on } \Gamma_{0} .\end{cases}
$$

Set $u_{2, i}:=X_{i}^{n_{1}}+V_{i} \in E_{i}$,

Step 2: The functions $u_{2, i}$ are the unique solutions of

$$
\begin{cases}L\left(u_{2, i}\right)=-h_{i}^{n_{2}}+f_{2} & \text { in } \Omega, \\ u_{2, i}=X_{i}^{n_{1}}+V_{i} & \text { on } \Gamma_{i}, \\ u_{2, i}=0 & \text { on } \Gamma-\Gamma_{i} \cup \Gamma_{0}, \\ u_{2, i}=0 & \text { on } \Gamma_{0} .\end{cases}
$$

Using the estimates established in step 1, we are ready to prove (3.10)-(3.11).

Proof of estimate (3.10): Since $\sigma\left(V_{i}\right) \cdot \vec{n}=\sigma\left(\tilde{Y}_{i}^{n_{2}}\right) \cdot \vec{n}$ on $\Gamma_{i}$, then we have for $g_{i}:=\sigma\left(V_{i}+X_{i}^{n_{1}}\right) \cdot \vec{n}$

$$
\left\|g_{i}-g_{p}^{\epsilon}\right\|_{0, \Gamma_{i}}=\left\|\sigma\left(V_{i}+X_{i}^{n_{1}}\right) \cdot \vec{n}-g_{p}^{\epsilon}\right\|_{0, \Gamma_{i}}=\left\|\sigma\left(\tilde{Y}_{i}^{n_{2}}+X_{i}^{n_{1}}\right) \cdot \vec{n}-\sigma\left(u_{\epsilon}^{p}\right) \cdot \vec{n}\right\|_{0, \Gamma_{i}} .
$$

Using (3.19) givesc

$$
\begin{aligned}
\left\|g_{i}-g_{p}^{\epsilon}\right\|_{0, \Gamma_{i}} & =\left\|\sigma\left(\tilde{Y}_{i}^{n_{2}}+Y_{i}^{n_{2}}+X_{i}^{n_{1}}\right) \cdot \vec{n}-\sigma\left(u_{\epsilon}^{p}\right) \cdot \vec{n}\right\|_{0, \Gamma_{i}} \\
& \leq\left\|\sigma\left(\tilde{Y}_{i}^{n_{2}}+Y_{i}^{n_{2}}+X_{i}^{n_{1}}\right) \cdot \vec{n}-\sigma\left(u_{\epsilon}^{p}\right) \cdot \vec{n}\right\|_{0, \Gamma-\Gamma_{0}} .
\end{aligned}
$$

For the fixed homogeneous Dirichlet condition on $\Gamma_{0}$, and according to the Banach isomorphism theorem, there exists a constant $C_{2}>0$ depending merely on $\Omega$ such that, using (3.22) and since we have $Y_{i}=Y_{i}^{n_{2}}+\tilde{Y}_{i}^{n_{2}}$

$$
\left\|g_{i}-g_{p}^{\epsilon}\right\|_{0, \Gamma_{i}} \leq\left\|\sigma\left(Y_{i}+X_{i}^{n_{1}}\right) \cdot \vec{n}-\sigma\left(u_{\epsilon}^{p}\right) \cdot \vec{n}\right\|_{0, \Gamma-\Gamma_{0}}
$$




$$
\leq C_{2}\left\|\nabla\left(Y_{i}+X_{i}^{n_{1}}\right)-\nabla u_{\epsilon}^{p}\right\|_{0, \Omega} \leq \frac{c_{t} \times C_{3} C_{2} \delta_{3}}{m} .
$$

If $\delta_{3}$ also fulfill condition

$$
\text { (c3): } \frac{c_{t} \times C_{3} C_{2} \delta_{3}}{m}<\frac{\delta_{1}}{m}
$$

where $\delta_{1}$ is the arbitrary small number assumed at the beginning of the proof then, estimate (3.10) is proved.

In order to prove estimate (3.11), we need to estimate the trace of $X_{i}^{n_{1}}+V_{i}$ on $\Gamma_{i}$.

1) Firstly remark, by using $(h 3)$, that

$$
V_{i}+X_{i}^{n_{1}}=0 \quad \text { on } \Gamma-\Gamma_{i} .
$$

2) For the fixed $-\left(h_{i}^{n_{2}}+F_{i}^{n_{1}}-f_{2}\right) \in L^{2}(\Omega), g^{\prime} \in L^{2}\left(\Gamma_{i}\right)$ and the homogeneous Dirichlet condition on $\Gamma_{0}$ in problems $\left(P_{6}\right)$ and $\left(P_{7}\right)$, the Banach isomorphism theorem ensures the existence of a constant $C_{4}>0$ depending merely on $\Omega$ such that

$$
\left\|\nabla V_{i}-\nabla \tilde{Y}_{i}^{n_{2}}\right\|_{0, \Omega} \leq C_{4}\left\|V_{i}-\tilde{Y}_{i}^{n_{2}}\right\|_{0, \Gamma-\Gamma_{i}}=C_{4}|| 0-\left(Y_{i}-y_{i}^{n_{2}}\right) \|_{0, \Gamma-\Gamma_{i}},
$$

hence, considering $(h 4)$ and using trace inequality, we have

$$
C_{4}\left\|0-\left(Y_{i}-y_{i}^{n_{2}}\right)\right\|_{0, \Gamma-\Gamma_{i}} \leq c_{t} C_{4}\left\|\nabla Y_{i}-\nabla Y_{i}^{n_{2}}\right\|_{0, \Omega} \leq c_{t} C_{4} \frac{\delta_{1}^{\prime}}{m},
$$

thus,

$$
\left\|\nabla V_{i}-\nabla \tilde{Y}_{i}^{n_{2}}\right\|_{0, \Omega} \leq c_{t} C_{4} \frac{\delta_{1}^{\prime}}{m}
$$

Using trace inequality and (3.23) we obtain

$$
\left\|V_{i}-0\right\|_{0, \Gamma_{i}} \leq\left\|V_{i}-\tilde{Y}_{i}^{n_{2}}\right\|_{0, \Gamma} \leq c_{t}\left\|\nabla V_{i}-\nabla \tilde{Y}_{i}^{n_{2}}\right\|_{0, \Omega} \leq c_{t}^{2} \times C_{4} \frac{\delta_{1}^{\prime}}{m} .
$$

Estimates (3.14) and (3.24) imply

$$
\begin{aligned}
& \left.\left\|V_{i}+X_{i}^{n_{1}}-u_{\epsilon}^{p}\right\|\right|_{0, \Gamma_{i}} \leq\left\|V_{i}-0\right\|_{0, \Gamma_{i}}+\left\|X_{i}^{n_{1}}-u_{\epsilon}^{p}\right\|_{0, \Gamma_{i}} \\
= & \left\|V_{i}-0\right\|_{0, \Gamma_{i}}+\left\|x_{i}^{n_{1}}-u_{\epsilon}^{p}\right\|_{0, \Gamma_{i}} \leq c_{t}^{2} \times C_{4} \frac{\delta_{1}^{\prime}}{m}+\frac{c_{t} \delta_{3}}{m} .
\end{aligned}
$$

Proof of estimate (3.11): Write $u_{2}^{\prime}:=\sum_{i} u_{2, i}=\zeta^{\prime}+\eta^{\prime}$ where $\zeta^{\prime}$ and $\eta^{\prime}$ are respectively the solutions of

$$
\left\{\begin{array} { l l } 
{ L ( \zeta ^ { \prime } ) = - \sum _ { i = 1 } ^ { m } ( h _ { i } ^ { n _ { 2 } } - f _ { 2 } ) } & { \text { in } \Omega , } \\
{ \zeta ^ { \prime } = 0 } & { \text { on } \Gamma _ { i } , } \\
{ \zeta ^ { \prime } = 0 } & { \text { on } \Gamma - \Gamma _ { i } . }
\end{array} \quad ( P _ { 8 } ) \quad \left\{\begin{array}{ll}
L\left(\eta^{\prime}\right)=0 & \text { in } \Omega, \\
\eta^{\prime}=\sum_{i=1}^{m} u_{2, i} & \text { on } \Gamma-\Gamma_{0}, \\
\eta^{\prime}=0 & \text { on } \Gamma_{0},
\end{array}\right.\right.
$$

and write $u_{2}=\zeta+\eta$ where $\zeta$ and $\eta$ are the solutions of 


$$
\left\{\begin{array} { l l } 
{ L ( \zeta ) = f _ { 2 } } & { \text { in } \Omega , } \\
{ \zeta = 0 } & { \text { on } \Gamma _ { i , } } \\
{ \zeta = 0 } & { \text { on } \Gamma - \Gamma _ { i } , }
\end{array} \quad ( P _ { 9 } ) \left\{\begin{array}{ll}
L(\eta)=0 & \text { in } \Omega, \\
\eta=u_{\epsilon}^{p} & \text { on } \Gamma-\Gamma_{0} \\
\eta=0 & \text { on } \Gamma_{0} .
\end{array}\right.\right.
$$

If we denote $c_{k}$ the constant in the Korn inequality then the weak form of the problem solved by $\zeta-\zeta^{\prime}$ yields

$$
\begin{aligned}
\left\|\nabla \zeta-\nabla \zeta^{\prime}\right\|_{0, \Omega} & \leq c_{k} d(\Omega)\left\|f_{2}+\sum_{i}\left(h_{i}^{n_{2}}-f_{2}\right)\right\|_{0, \Omega} \\
& \leq 2 d(\Omega)\left(\left\|f_{2}\right\|_{0, \Omega}+\sum_{i}\left\|h_{i}^{n_{2}}\right\|_{0, \Omega}+\sum_{i}\left\|f_{2}\right\|_{0, \Omega}\right) .
\end{aligned}
$$

Using (iv) of Lemmas 3.1 and (3.21), we have

$$
\left\|\nabla \zeta-\nabla \zeta^{\prime}\right\|_{0, \Omega} \leq d(\Omega)\left(\frac{\delta_{0}}{2 m}+\delta_{0}+\frac{\delta_{0}}{2}\right) .
$$

On the other hand, the Banach isomorphism theorem ensures, for the null source term and the Dirichlet homogeneous condition on $\Gamma_{0}$ in problems $\left(P_{8}\right)$ and $\left(P_{9}\right)$, that there exists a constant $C_{5}>0$ such that

$$
\begin{aligned}
\left\|\nabla \eta-\nabla \eta^{\prime}\right\|_{0, \Omega} & \leq C_{5}\left\|\eta-\eta^{\prime}\right\|_{0, \Gamma-\Gamma_{0}}=C_{5}|| u_{\epsilon}^{p}-\sum_{i=1}^{m} u_{2, i} \|_{0, \Gamma-\Gamma_{0}} \\
& \leq C_{5} \sum_{i=1}^{m}\left\|u_{\epsilon}^{p}-\sum_{i=1}^{m} u_{2, i}\right\|_{0, \Gamma_{i}}=C_{5} \sum_{i=1}^{m}\left\|u_{\epsilon}^{p}-u_{2, i}\right\|_{0, \Gamma_{i}} .
\end{aligned}
$$

Applying estimate (3.25) with $u_{2, i}=V_{i}+X_{i}^{n_{1}}$

$$
\left\|\nabla \eta-\nabla \eta^{\prime}\right\|_{0, \Omega} \leq C_{5}\left(c_{t}^{2} \times C_{4} \delta_{1}^{\prime}+c_{t} \delta_{3}\right) .
$$

Combining (3.26) and (3.27), we obtain using triangular inequality

$$
\begin{aligned}
\left\|\nabla u_{2}-\nabla u_{2}^{\prime}\right\|_{0, \Omega} & \leq\left\|\nabla \zeta-\nabla \zeta^{\prime}\right\|+\left\|\nabla \eta-\nabla \eta^{\prime}\right\|_{0, \Omega} \\
& \leq d(\Omega)\left(\frac{\delta_{0}}{2 m}+\delta_{0}+\frac{\delta_{0}}{2}\right)+C_{5}\left(c_{t}^{2} \times C_{4} \delta_{1}^{\prime}+c_{t} \delta_{3}\right) .
\end{aligned}
$$

Finally, if $\delta_{0}, \delta_{3}$ and $\delta_{1}^{\prime}$ are subject to fulfill the condition

$$
(c 4): \quad d(\Omega)\left(\frac{\delta_{0}}{2 m}+\delta_{0}+\frac{\delta_{0}}{2}\right)+C_{5}\left(c_{t}^{2} \times C_{4} \delta_{1}^{\prime}+c_{t} \delta_{3}\right)<\delta_{2}
$$

where $\delta_{2}$ is the arbitrary small number assumed at the beginning of the proof then,

$$
\left\|\nabla u_{\epsilon}^{p}-\nabla\left(u_{1}+u_{2}^{\prime}\right)\right\|_{0, \Omega} \leq \delta_{2} .
$$

This proves (3.28). 
Remark 3.1. 1) Let $g_{p}^{\epsilon}$ such as defined in (3.4), we can easily show that

$$
\left\|g_{p}^{\epsilon}\right\|_{0, \Gamma-\Gamma_{0}}^{2} \leq\|g\|_{0, \Gamma-\Gamma_{0}}^{2}+\delta_{6}^{2}
$$

where $\delta_{6}$ is an arbitrary small positive real number. Indeed, write using triangular inequality

$$
\begin{aligned}
& \left.\left|\| g_{p}^{\epsilon}\right|\right|_{0, \Gamma-\Gamma_{0}} ^{2}-\int_{\Gamma-\Gamma_{0}} g g_{p}^{\epsilon} \mathrm{d} \sigma \mid \\
= & \left|<g_{p}^{\epsilon}, g_{p}^{\epsilon}\right\rangle_{\left[H^{\frac{1}{2}}\left(\Gamma-\Gamma_{0}\right)\right]^{\prime}, H^{\frac{1}{2}}\left(\Gamma-\Gamma_{0}\right)}-<g, g_{p}^{\epsilon}>_{\left[H^{\frac{1}{2}}\left(\Gamma-\Gamma_{0}\right)\right]^{\prime}, H^{\frac{1}{2}}\left(\Gamma-\Gamma_{0}\right)} \mid \\
\leq & \left|<g_{p}^{\epsilon}-g, g_{p}^{\epsilon}>_{\left[H^{\frac{1}{2}}\left(\Gamma-\Gamma_{0}\right)\right]^{\prime}, H^{\frac{1}{2}}\left(\Gamma-\Gamma_{0}\right)}\right| \\
\leq & \left\|g_{p}^{\epsilon}-g\right\|_{\left[H^{\frac{1}{2}}\left(\Gamma-\Gamma_{0}\right)\right]^{\prime}}\left\|g_{p}^{\epsilon}\right\|_{\frac{1}{2}, \Gamma-\Gamma_{0}} \\
\leq & \left(\left\|g_{p}^{\epsilon}-\left.g^{\epsilon}\right|_{\left[H^{\frac{1}{2}}\right]^{\prime}}+\right\| g^{\epsilon}-g \|_{\left[H^{\frac{1}{2}}\right]^{\prime}}\right)|| g_{p}^{\epsilon} \|_{\frac{1}{2}, \Gamma-\Gamma_{0}} .
\end{aligned}
$$

Since, following estimate $(i i)$ of (3.7), $g_{p}^{\epsilon}$ converges in $\left[H^{\frac{1}{2}}\left(\Gamma-\Gamma_{0}\right)\right]^{\prime}$ to $g^{\epsilon}$ i.e. weakly in $H^{\frac{1}{2}}\left(\Gamma-\Gamma_{0}\right)$ hence, there exists $C^{\prime}>0$ such that $\left\|g_{p}^{\epsilon}\right\|_{\frac{1}{2}, \Gamma-\Gamma_{0}} \leq C^{\prime}$ for all $p \in \mathbb{N}$. Thus,

$$
\left\|g_{p}^{\epsilon}\right\|_{0, \Gamma-\Gamma_{0}}^{2}-\int_{\Gamma-\Gamma_{0}} g g_{p}^{\epsilon} \mathrm{d} \sigma \leq C^{\prime}\left(\left\|g_{p}^{\epsilon}-g^{\epsilon}\right\|_{\left[H^{\frac{1}{2}}\left(\Gamma-\Gamma_{0}\right)\right]^{\prime}}+\left\|g^{\epsilon}-g\right\|_{\left[H^{\frac{1}{2}}\left(\Gamma-\Gamma_{0}\right)\right]^{\prime}}\right) .
$$

Using (i) of Remark 2.2 and estimates (ii) of (3.7) and for an adequate choice of $\beta$, the parameters $p$ and $\epsilon$ can be chosen such that

$$
\left\|g_{p}^{\epsilon}\right\|_{0, \Gamma-\Gamma_{0}}^{2}-\int_{\Gamma-\Gamma_{0}} g g_{p}^{\epsilon} \mathrm{d} \sigma \leq 2 \beta C^{\prime}
$$

and thus, Cauchy-Schwarz yields

$$
\left\|g_{p}^{\epsilon}||_{0, \Gamma-\Gamma_{0}}^{2} \leq \int_{\Gamma-\Gamma_{0}} g g_{p}^{\epsilon} \mathrm{d} \sigma+2 \beta C^{\prime} \leq\right\| g\left\|_{0, \Gamma-\Gamma_{0}}\right\| g_{p}^{\epsilon} \|_{0, \Gamma-\Gamma_{0}}+2 \beta C^{\prime} .
$$

Young inequality on the other hand gives us

$$
\left\|g_{p}^{\epsilon}\right\|_{0, \Gamma-\Gamma_{0}}^{2} \leq \frac{1}{2}\|g\|_{0, \Gamma-\Gamma_{0}}^{2}+\frac{1}{2}\left\|g_{p}^{\epsilon}\right\|_{0, \Gamma-\Gamma_{0}}^{2}+2 \beta C^{\prime}
$$

and hence, there exists $p$ such that $g_{p}^{\epsilon}$ satisfy

$$
\left\|g_{p}^{\epsilon}\right\|_{0, \Gamma-\Gamma_{0}}^{2} \leq\|g\|_{0, \Gamma-\Gamma_{0}}^{2}+4 \beta C^{\prime}
$$

Pose $\delta_{6}^{2}:=4 \beta C^{\prime}$. Since, following ( $i$ ) of Remark 2.2 and (3.7), $\beta$ is arbitrary small, then the same holds with $\delta_{6}$. 
2) On the other hand, if $u_{2, i}$ and $h_{i}^{n_{2}}$ are respectively such as defined in Lemma 3.2 and (3.20) then

$$
\left\|L\left(u_{2, i}\right)\right\|_{0, \Omega}=\left\|h_{i}^{n_{2}}+f_{2}\right\|_{0, \Omega} \leq\left\|h_{i}^{n_{2}}\right\|_{0, \Omega}+\left\|f_{2}\right\|_{0, \Omega} \leq \frac{\delta_{0}}{m}+\frac{\delta_{0}}{2 m}=: \tau,
$$

where $\delta_{0}$ is arbitrary small and satisfy $(c 4)$ and thus, $\tau$ is also arbitrary small.

We introduce some useful lemmas, which will play important roles in the proof of Theorem 1.1.

\subsection{Extension of the functions $u_{2, i}$}

Since we are looking for explicit estimates, we should use Poincaré, trace and Korn's inequalities relatively to suitable geometric configurations i.e. for which they are explicitly formulated. The configuration that best fits our polygonal convex domain $\Omega$ is the half-plane $\mathbb{R}^{2+}$ containing the domain $\Omega$ for Korn's inequality, the square $S_{d}$ with edge's length equal to $d(\Omega)$ for the Poincaré inequality and with edge $\Gamma_{i}$ for the trace inequality. These squares are taken to be subsets of the half-plane containing $\Omega$. Thus we determine these constants thanks to results available for this type of domains. The need to consider the functions in and outside the domain $\Omega$ suggests to extend by zero the functions $u_{2, i}$ outside the convex domain $\Omega$. The definition of the functions $u_{2, i}$ is adapted to make such an extension.

Let $u_{2, i}$ be such as defined in Lemma 3.2. We consider for $i, 1 \leq i \leq m$, the extension by zero of $u_{2, i}$ from the convex domain $\Omega$ to the half-plane $\mathbb{R}^{2+}$ containing $\Omega$ such that $\Gamma_{i} \subset \partial \mathbb{R}^{2+}$.

$$
\tilde{u}_{2, i}= \begin{cases}u_{2, i}, & \text { a.e. } x \in \bar{\Omega}, \\ 0, & x \in \mathbb{R}^{2+}-\bar{\Omega} .\end{cases}
$$

We assert that $\tilde{u}_{2, i} \in H^{1}\left(\mathbb{R}^{2+}\right)$, this is due essentially to definition (1.9), this definition implies the existence of an open neighborhood $V_{i}$ of the vertex $i$ such that $u_{2, i}$ vanish on $V_{i} \cap \Gamma$. Thus, we have, obviously, the following

$$
\left\|\left.\partial_{x_{i}} \tilde{u}_{2, i}\right|_{0, \mathbb{R}^{2+}}=|| \partial_{x_{i}} \tilde{u}_{2, i}\right\|_{0, \Omega}=\left.|| \partial_{x_{i}} u_{2, i}\right|_{0, \Omega}
$$

One can easily see that the resulting extend functions $\tilde{u}_{2, i}$ are still in the Sobolev space $H^{1}\left(\mathbb{R}^{2+}\right)$. Indeed, thanks the definition of these function, there will be no jump when passing to the distributional derivative.

The following inequalities are established for the extended $H^{1}$ regular functions defined on a square containing the convex polygonal domain $\Omega$. 


\subsection{Explicit constant in the Poincaré inequality}

We show in the following lemma that the function $u_{2, i} \in E_{i}$ satisfy the Poincare inequality for which we determine explicitly the constant.

Lemma 3.3. For all $i, 0 \leq i \leq m$, the function $u_{2, i}$ satisfy:

$$
\left\|u_{2, i}\right\|_{0, \Omega} \leq d(\Omega)\left\|\nabla u_{2, i}\right\|_{0, \Omega},
$$

the constant $d(\Omega)$ means the diameter of $\Omega$.

Proof. We establish Poincare inequality for one of the two components $u_{2, i^{\prime}}^{l} l=1,2$, the same estimate hold with the other. Note $a b c d$ the square $S_{d}$, with edge's length equal to $d(\Omega)$ and subset of the half-plane containing $\Omega$, such that $a=\left(a_{1}, a_{2}\right), b=\left(b_{1}, b_{2}\right), c=\left(c_{1}, c_{2}\right)$ and $d=\left(d_{1}, d_{2}\right)$ and such that $\Gamma_{i} \subset S_{i}:=[c, d]$; so $\tilde{u}_{2, i}^{l}=0$ on $\partial S_{d}-\Gamma_{i}$.

Since $\tilde{u}_{2, i}^{l}$ is absolutely continuous on the lines parallel to the coordinate axis, then applying the fundamental theorem of calculus to $\tilde{u}_{1, i}^{n, l}$ on $S_{d}$ for $l=1,2$, we have for all $\left(x_{1}, x_{2}\right) \in\left[a_{1}, d_{1}\right] \times\left[a_{2}, b_{2}\right]$

$$
\tilde{u}_{2, i}^{l}\left(x_{1}, x_{2}\right)=\int_{a_{1}}^{x_{1}} \partial_{x_{1}} \tilde{u}_{2, i}^{l}\left(s, x_{2}\right) \mathrm{d} s+\tilde{u}_{2, i}^{l}\left(a_{1}, x_{2}\right) .
$$

Since $\left(a_{1}, x_{2}\right) \in \partial S_{d}-\Gamma_{i}$, then $\forall\left(x_{1}, x_{2}\right) \in\left[a_{1}, d_{1}\right] \times\left[a_{2}, b_{2}\right]$

$$
\tilde{u}_{2, i}^{l}\left(x_{1}, x_{2}\right)=\int_{a_{1}}^{x_{1}} \partial_{x_{1}} \tilde{u}_{2, i}^{l}\left(s, x_{2}\right) \mathrm{d} s .
$$

Using Cauchy-Schwarz inequality $\forall\left(x_{1}, x_{2}\right) \in\left[a_{1}, d_{1}\right] \times\left[a_{2}, b_{2}\right]$

$$
\left|\tilde{u}_{2, i}^{l}\left(x_{1}, x_{2}\right)\right| \leq\left|x_{1}-a\right|^{\frac{1}{2}}\left(\int_{a_{1}}^{x_{1}}\left|\partial_{x_{1}} \tilde{u}_{2, i}^{l}\left(s, x_{2}\right)\right|^{2} \mathrm{~d} s\right)^{\frac{1}{2}} .
$$

Taking the square of the two hand sides of this inequality and using the fact $\left|x_{1}-a\right| \leq$ $d(\Omega): \forall\left(x_{1}, x_{2}\right) \in\left[a_{1}, d_{1}\right] \times\left[a_{2}, b_{2}\right]$ yields

$$
\left|\tilde{u}_{2, i}^{l}\left(x_{1}, x_{2}\right)\right|^{2} \leq\left|x_{1}-a\right| \int_{a_{1}}^{x_{1}}\left|\partial_{x_{1}} \tilde{u}_{2, i}^{l}\left(s, x_{2}\right)\right|^{2} \mathrm{~d} s \leq d(\Omega) \int_{a_{1}}^{d_{1}}\left|\partial_{x_{1}} \tilde{u}_{2, i}^{l}\left(s, x_{2}\right)\right|^{2} \mathrm{~d} s .
$$

Integrating on $S_{d}$ with respect to the variables $x_{1}$ and $x_{2}$ :

$$
\begin{aligned}
\left\|\tilde{u}_{2, i}^{l}\right\|_{0, S_{d}}^{2} & =\int_{a_{2}}^{b_{2}} \int_{a_{1}}^{d_{1}}\left|\tilde{u}_{2, i}^{l}\left(x_{1}, x_{2}\right)\right|^{2} \mathrm{~d} x_{1} \mathrm{~d} x_{2} \\
& \leq d(\Omega) \int_{a_{2}}^{b_{2}} \int_{a_{1}}^{d_{1}} \int_{a_{1}}^{d_{1}}\left|\partial_{x} \tilde{u}_{2, i}^{l}\left(s, x_{2}\right)\right|^{2} \mathrm{~d} s \mathrm{~d} x_{1} \mathrm{~d} x_{2} \\
& \leq d^{2}(\Omega) \iint_{S_{d}}\left|\partial_{x} \tilde{u}_{2, i}^{l}\left(s, x_{2}\right)\right|^{2} \mathrm{~d} s \mathrm{~d} x_{2} .
\end{aligned}
$$


According to definition (3.30) and by considering (3.31) we get

$$
\left\|u_{2, i}^{l}\right\|_{0, \Omega}^{2} \leq d^{2}(\Omega)\left\|\nabla u_{2, i}^{l}\right\|_{0, \Omega}^{2} .
$$

We infer that

$$
\begin{gathered}
\left\|u_{2, i}\right\|_{0, \Omega}^{2}=\left\|u_{2, i}^{1}\right\|_{0, \Omega}^{2}+\left\|u_{2, i}^{2}\right\|_{0, \Omega}^{2} \\
\leq d^{2}(\Omega)\left(\left\|\nabla u_{2, i}^{1}\right\|_{0, \Omega}^{2}+\left\|\nabla u_{2, i}^{2}\right\|_{0, \Omega}^{2}\right)=d^{2}(\Omega)\left\|\nabla u_{2, i}\right\|_{0, \Omega}^{2} .
\end{gathered}
$$

This completes the proof of the lemma.

\subsection{Explicit bound for the trace of $u_{2, i}$ on $\Gamma_{i}$}

Using mainly the inequality of Poincare stated in Lemma 3.3 and the trace inequality for $u_{2, i}$ on the edge $\Gamma_{i}$ of a the parallelogram $S_{\Gamma_{i}}$, see ([9, Lemma 4.2]) as well as ([10, Remark 3.3],) and the reference therein, one establishes an explicit bound for the trace of the function $u_{2, i}$ on $\Gamma_{i}$.

Lemma 3.4. For all $i$, the function $u_{2, i}$ defined in Lemma 3.2 satisfy:

$$
\left\|\left.u_{2, i}\right|_{0, \Gamma_{i}} \leq 16\left|\Gamma_{i}\right||| g_{i}\right\|_{0, \Gamma_{i}}+\delta_{9}
$$

where $\delta_{9}$ is an arbitrary small real positive number.

Proof. Let $\tilde{u}_{2, i}$ be defined on the square $S_{\Gamma_{i}}$ with edge $\Gamma_{i}$ such that $S_{\Gamma_{i}}$ is a subset of the halfplane $\mathbb{R}^{2+}$ containing $\Omega$. One should notice that $\tilde{u}_{2, i}$ is not necessarily zero on $\partial S_{\Gamma_{i}}-\Gamma_{i}$. Let us write

$$
\tilde{u}_{2, i}=\alpha+\varrho,
$$

this decomposition is made similarly to that presented in Lemma 3.2, i.e., such that on one hand

$$
\|L(\alpha)\|_{0, S_{\Gamma_{i}}} \text { and }\left\|\sigma(\alpha) \cdot \vec{n}-g_{i}\right\|_{0, \Gamma_{i}} \text { are small enough }
$$

and on the other hand, $\alpha, \varrho$ vanish respectively on $\partial S_{\Gamma_{i}}-\Gamma_{i}, \Gamma_{i}$. We prove (3.33) for the trace of the function $\alpha$, the same estimate holds for the trace of $u_{2, i}$ since $\alpha$ and $u_{2, i}$ coincide on $\Gamma_{i}$. Decompose $\alpha:=\alpha_{1}+\alpha_{2}$ in the following way

$$
\left\{\begin{array} { l l } 
{ L ( \alpha _ { 1 } ) = L ( \alpha ) } & { \text { in } S _ { \Gamma _ { i } , } , } \\
{ \sigma ( \alpha _ { 1 } ) \cdot \vec { n } = 0 } & { \text { on } \Gamma _ { i } , } \\
{ \alpha _ { 1 } = 0 } & { \text { on } \partial S _ { \Gamma _ { i } } - \Gamma _ { i } . }
\end{array} \quad \left\{\begin{array}{ll}
L\left(\alpha_{2}\right)=0 & \text { in } S_{\Gamma_{i}}, \\
\sigma\left(\alpha_{2}\right) \cdot \vec{n}=\sigma(\alpha) \cdot \vec{n} & \text { on } \Gamma_{i}, \\
\alpha_{2}=0 & \text { on } \partial S_{\Gamma_{i}}-\Gamma_{i} .
\end{array}\right.\right.
$$


Applying the inequality of trace on the boundary $\Gamma_{i}$ of the the square $S_{\Gamma_{i}}$, which is a parallelogram, see ([9], Lemma 4.2), it yields for both $k=1,2$

$$
\left\|\alpha_{k}\right\|_{0, \Gamma_{i}}^{2} \leq 2 \frac{\left|\Gamma_{i}\right|}{\left|\Gamma_{i}\right|^{2}}|| \alpha_{k}\left\|_{0, S_{\Gamma_{i}}}^{2}+2 \frac{\left|\Gamma_{i}\right|^{2}}{\left|\Gamma_{i}\right|}\right\| \nabla \alpha_{k} \|_{0, S_{\Gamma_{i}}}^{2},
$$

simplifying,

$$
\left\|\alpha_{k}\right\|_{0, \Gamma_{i}}^{2} \leq 2 \frac{1}{\left|\Gamma_{i}\right|}\left\|\alpha_{k}\right\|_{0, S_{\Gamma_{i}}}^{2}+2\left|\Gamma_{i}\right| \mid \nabla \alpha_{k} \|_{0, S_{\Gamma_{i}}}^{2}
$$

using estimate (3.32) for the case where $\Omega$ is the square $S_{\Gamma_{i}}$, with $d\left(S_{\Gamma_{i}}\right)=\sqrt{2}\left|\Gamma_{i}\right|$, yileds

$$
\left\|\alpha_{k}\right\|_{0, \Gamma_{i}}^{2} \leq 2 \frac{2\left|\Gamma_{i}\right|^{2}}{4\left|\Gamma_{i}\right|}|| \nabla \alpha_{k}\left\|_{0, S_{\Gamma_{i}}}^{2}+2\left|\Gamma_{i}\right|\left|\nabla \alpha_{k}\left\|_{0, S_{\Gamma_{i}}}^{2} \leq 3\left|\Gamma_{i}\right||| \nabla \alpha_{k}\right\|_{0, S_{\Gamma_{i}}}^{2}\right.\right.
$$

Applying Korn's inequality for the extended function $\tilde{\alpha}$ defined on the half-plane $\mathbb{R}^{2+}$ containing $S_{\Gamma_{i}}$, for which case Korn constant equals $\frac{1}{2}$, estimate (3.35) becomes

$$
\left\|\alpha_{k}\right\|_{0, \Gamma_{i}}^{2} \leq 12\left|\Gamma_{i}\right||| \varepsilon\left(\alpha_{k}\right) \|_{0, S_{\Gamma_{i}}}^{2}
$$

Since $\alpha_{1}$ and $\alpha_{2}$ satisfy respectively

$$
\left\|\varepsilon\left(\alpha_{1}\right)\right\|_{0, S_{\Gamma_{i}}}^{2}=\int_{S_{\Gamma_{i}}} L(\alpha) \alpha_{1} \mathrm{~d} x \text { and }\left\|\varepsilon\left(\alpha_{2}\right)\right\|_{0, S_{\Gamma_{i}}}^{2}=\int_{\Gamma_{i}} \sigma(\alpha) \cdot \vec{n} \alpha_{2} \mathrm{~d} \sigma,
$$

then, using Cauchy-Schwarz, this leads to estimates

$$
\left\|\varepsilon\left(\alpha_{1}\right)\right\|_{0, S_{\Gamma_{i}}} \leq 2 \sqrt{2}\left|\Gamma_{i}\right|\|L(\alpha)\|_{0, S_{\Gamma_{i}}} \text { and } \quad\left\|\varepsilon\left(\alpha_{2}\right)\right\|_{0, S_{\Gamma_{i}}}^{2} \leq\|\sigma(\alpha) \cdot \vec{n}\|_{0, \Gamma_{i}}|| \alpha_{2} \|_{0, \Gamma_{i}}
$$

Using these last estimates, (3.36) becomes

$$
\left\|\alpha_{1}\right\|_{0, \Gamma_{i}}^{2} \leq 12 \times 8\left|\Gamma_{i}\right|^{2}\|L(\alpha)\|_{0, S_{\Gamma_{i}},}^{2} \quad\left\|\alpha_{2}\right\|_{0, \Gamma_{i}}^{2} \leq\left. 12\left|\Gamma_{i}\right||| \sigma(\alpha) \cdot \vec{n}||_{0, \Gamma_{i}}|| \alpha_{2}\right|_{0, \Gamma_{i}} .
$$

Using (3.29) and assumption (3.34), we can find $\delta_{5}$ and $\delta_{6}$ small enough such that

$$
\left.\left\|\left.\alpha_{1}\right|_{0, \Gamma_{i}} \leq \delta_{5}, \quad\right\| \alpha_{2}\right|_{0, \Gamma_{i}} \leq\left. 12\left|\Gamma_{i}\right||| g_{i}\right|_{0, \Gamma_{i}}+\delta_{6},
$$

for all $i$. Hence, combining these two estimates, we obtain

$$
\left\|u_{2, i}||_{0, \Gamma_{i}} \leq\right\| \alpha_{1}\left\|_{0, \Gamma_{i}}+|| \alpha_{2}\right\|_{0, \Gamma_{i}} \leq \delta_{9}+16\left|\Gamma_{i}\right||| g_{i} \|_{0, \Gamma_{i}}
$$

with $\delta_{9}=\delta_{5}+\delta_{6}$ arbitrary small. 


\section{Proof of Theorem 1.1}

We are ready now to present a proof of the main theorem. It uses, principally, Lemmas 3.1 and 3.2.

Proof. Step 1: Let $u_{1}$ be as defined in Lemma 3.1, it is easy to check that it satisfies

$$
2 \mu \int_{\Omega} \varepsilon\left(u_{1}\right) \varepsilon(v) \mathrm{d} x+\lambda \int_{\Omega} \operatorname{div} u_{1} \operatorname{div} v \mathrm{~d} x=\int_{\Omega} f_{1} v \mathrm{~d} x
$$

for all $v \in H_{0}^{1}(\Omega)$. Choose $v=u_{1}$ and use Cauchy-Schwarz inequality

$$
2 \mu\left\|\varepsilon\left(u_{1}\right)\right\|_{0, \Omega}^{2}+\lambda\left\|\operatorname{div} u_{1}\right\|_{0, \Omega}^{2} \leq\left\|f_{1}\right\|_{0, \Omega}\left\|u_{1}\right\|_{0, \Omega} .
$$

Using Korn's inequality relatively to the case of homogeneous Dirichlet condition on one hand, and Poincaré inequality on the other hand, yield

$$
\frac{\mu}{2}\left\|\nabla u_{1}\right\|_{0, \Omega}^{2} \leq\left\|f_{1}\right\|_{0, \Omega} \frac{d(\Omega)}{2}\left\|\nabla u_{1}\right\|_{0, \Omega}
$$

using (iv) of Lemma 3.1, we obtain

$$
\mu\left\|\nabla u_{1}\right\|_{0, \Omega} \leq d(\Omega)\left\|f^{p}\right\|_{0, \Omega}+d(\Omega) \frac{\delta_{0}}{m} .
$$

Fix $i, 1 \leq i \leq m$. Let $u_{2, i}$ be such as defined in Lemma 3.2, it is easy to check that $u_{2, i}$ satisfies

$$
2 \mu \int_{\Omega} \varepsilon\left(u_{2, i}\right) \varepsilon(v) \mathrm{d} x+\lambda \int_{\Omega} \operatorname{div} u_{2, i} \operatorname{div} v \mathrm{~d} x=\int_{\Omega} L\left(u_{2, i}\right) v \mathrm{~d} x+\int_{\Gamma-\Gamma_{0}} g_{i} v \mathrm{~d} \sigma
$$

for all $v \in V$. Choose $v=u_{2, i}$ and use Cauchy-Schwarz to obtain

$$
2 \mu\left\|\varepsilon\left(u_{2, i}\right)\right\|_{0, \Omega}^{2}+\lambda\left\|\operatorname{div} u_{2, i}\right\|_{0, \Omega}^{2} \leq\left.\left\|L\left(u_{2, i}\right)\right\|_{0, \Omega}|| u_{2, i}\right|_{0, \Omega}+\left\|g_{i}\right\|_{0, \Gamma_{i}}|| u_{2, i} \|_{0, \Gamma_{i}},
$$

using estimate (3.32)

$$
2 \mu\left\|\varepsilon\left(u_{2, i}\right)\right\|_{0, \Omega}^{2} \leq\left.\left\|L\left(u_{2, i}\right)\right\|_{0, \Omega} d(\Omega)|| \nabla u_{2, i}\right|_{0, \Omega}+\left\|g_{i}\right\|_{0, \Gamma_{i}}|| u_{2, i} \|_{0, \Gamma_{i}} .
$$

On the other hand, there exists constants $c_{k}, c_{p}$ and $c_{t}$ such that

$$
\left.2 \mu c_{k}|| \nabla u_{2, i}\right|_{0, \Omega} \leq\left. c_{p}|| L\left(u_{2, i}\right)\right|_{0, \Omega}+c_{t}|| g_{i} \|_{0, \Gamma_{i}} .
$$

Consequently,

$$
\begin{aligned}
& 2 \mu\left\|\varepsilon\left(u_{2, i}\right)\right\|_{0, \Omega}^{2} \\
\leq & d(\Omega)\left\|L\left(u_{2, i}\right)\right\|_{0, \Omega} \frac{1}{2 \mu c_{k}}\left(c_{p}\left\|L\left(u_{2, i}\right)\right\|_{0, \Omega}+c_{t}\left\|g_{i}\right\|_{0, \Gamma_{i}}\right)+\left\|g_{i}\right\|_{0, \Gamma_{i}}\left\|u_{2, i}\right\|_{0, \Gamma_{i}}
\end{aligned}
$$


since, following (3.10) and (3.28), $\left\|g_{i}\right\|_{0, \Omega}$ are uniformly bounded with respect to $\epsilon$ then, using (3.29)

$$
\begin{aligned}
2 \mu\left\|\varepsilon\left(u_{2, i}\right)\right\|_{0, \Omega}^{2} & \leq d(\Omega) \tau \frac{1}{2 \mu c_{k}}\left(c_{p} \tau+c_{t}|| g_{i} \|_{0, \Gamma_{i}}\right)+\left\|g_{i}\right\|_{0, \Gamma_{i}}\left\|u_{2, i}\right\|_{0, \Gamma_{i}} \\
& \leq d(\Omega) \frac{\tau}{2 \mu c_{k}}\left(c_{p} \tau+c_{t}\left(\|g\|_{0, \Gamma-\Gamma_{0}}+\delta_{6}+\frac{\delta_{1}}{m}\right)\right)+\left\|g_{i}\right\|_{0, \Gamma_{i}}|| u_{2, i} \|_{0, \Gamma_{i}} ;
\end{aligned}
$$

pose

$$
\delta_{7}^{2}:=d(\Omega) \frac{\tau}{2 \mu c_{k}}\left(c_{p} \tau+c_{t}\left(\|g\|_{0, \Gamma-\Gamma_{0}}+\delta_{6}+\frac{\delta_{1}}{m}\right)\right),
$$

it can be made, thanks to estimate (3.29), as small as desired. Applying (3.33), (3.10) and (3.28), estimate (4.3) becomes

$$
\begin{aligned}
& 2 \mu\left\|\varepsilon\left(u_{2, i}\right)\right\|_{0, \Omega}^{2} \leq \delta_{7}^{2}+16\left|\Gamma_{i}\right|\left\|g_{i}\right\|_{0, \Gamma_{i}}^{2}+\delta_{9}|| g_{i} \|_{0, \Gamma_{i}} \\
\leq & \delta_{7}^{2}+16\left|\Gamma_{i}\right|\left\|g_{i}\right\|_{0, \Gamma_{i}}^{2}+\delta_{9}\left(\frac{\delta_{1}}{m}+\delta_{6}+\|g\|_{0, \Gamma_{i}}\right) \leq \frac{\delta_{10}^{2}}{m^{2}}+16\left|\Gamma_{i}\right|\left\|g_{i}\right\|_{0, \Gamma_{i}}^{2}
\end{aligned}
$$

where $\frac{\delta_{10}^{2}}{m^{2}}:=\delta_{7}^{2}+\delta_{9}\left(\frac{\delta_{1}}{m}+\delta_{6}+\|g\|_{0, \Gamma_{i}}\right)$. Then by simplifying,

$$
\sqrt{2 \mu}\left\|\varepsilon\left(u_{2, i}\right)\right\|_{0, \Omega} \leq \frac{\delta_{10}}{m}+4\left|\Gamma_{i}\right|^{\frac{1}{2}}|| g_{i} \|_{0, \Gamma_{i}} .
$$

One should notice that, from the definition of the constants $\delta_{6}, \delta_{7}$ and $\delta_{9}$, the constant $\delta_{10}$ can be made as small as desired.

Step 2: Since the deformation $\varepsilon\left(u_{2, i}\right)$ is a linear application with respect to the first derivatives of $u_{2, i}$ then, with the same notations as in (3.30) and by using (3.31), we have

$$
\left\|\varepsilon\left(\tilde{u}_{2, i}\right)\right\|_{0, \mathbb{R}^{2+}}=\left\|\varepsilon\left(\tilde{u}_{2, i}\right)\right\|_{0, \Omega}=\left\|\varepsilon\left(u_{2, i}\right)\right\|_{0, \Omega_{i}} .
$$

Applying the estimate stated in ([11], Corollary 1.2.2) to $\tilde{u}_{2, i}$ gives

$$
\frac{1}{2} \times\left\|\left.\nabla u_{2, i}\right|_{0, \Omega}=\frac{1}{2} \times\right\| \nabla \tilde{u}_{2, i}\left\|_{0, \mathbb{R}^{2+}} \leq\right\| \varepsilon\left(\tilde{u}_{2, i}\right) \|_{0, \mathbb{R}^{2+}} .
$$

Thus, (4.4) becomes

$$
\frac{\sqrt{\mu}}{\sqrt{2}}\left\|\left.\nabla u_{2, i}\right|_{0, \Omega} \leq \frac{\delta_{10}}{m}+4\left|\Gamma_{i}\right|^{\frac{1}{2}}|| g_{i}\right\|_{0, \Gamma_{i}} .
$$

Using young inequality yields

$$
\frac{\sqrt{\mu}}{\sqrt{2}}\left\|\nabla u_{2, i}\right\|_{0, \Omega} \leq \frac{\delta_{10}}{m}+2\left(\left|\Gamma_{i}\right|+|| g_{i} \|_{0, \Gamma_{i}}^{2}\right) .
$$

Using approximation result (3.10), we have

$$
\frac{\sqrt{\mu}}{\sqrt{2}}\left\|\nabla u_{2, i}\right\|_{0, \Omega} \leq \frac{\delta_{10}}{m}+4\left(\left|\Gamma_{i}\right|+|| g_{p}^{\epsilon} \|_{0, \Gamma_{i}}^{2}+\frac{\delta_{1}^{2}}{m^{2}}\right) .
$$


Summing over $i=\overline{1, m}$ we obtain using triangular inequality

$$
\left.\frac{\sqrt{\mu}}{\sqrt{2}}|| \nabla u_{2}^{\prime}\right|_{0, \Omega} \leq \frac{\sqrt{\mu}}{\sqrt{2}} \sum_{i}|| \nabla u_{2, i}\left\|_{0, \Omega} \leq \delta_{10}+4|\Gamma|+4|| g_{p}^{\epsilon}\right\|_{0, \Gamma}^{2}+4 \delta_{1}^{2} .
$$

Finally, combining (4.1) and (4.5), we get

$$
\sqrt{\mu}|| \nabla\left(u_{1}+u_{2}^{\prime}\right)\left\|_{0, \Omega} \leq \frac{\delta_{0}}{m} d(\Omega)+\sqrt{2} \delta_{10}+4 \delta_{1}^{2}+d(\Omega)\right\| f^{p} \|_{0, \Omega}+8\left(|\Gamma|+\left\|g_{p}^{\epsilon}\right\|_{0, \Gamma}^{2}\right) .
$$

Considering (3.11) and posing $\delta_{11}:=\frac{\delta_{0}}{m} d(\Omega)+\sqrt{2} \delta_{10}+4 \delta_{1}^{2}$, we have

$$
\sqrt{\mu}|| \nabla u_{\epsilon}^{p}\left\|_{0, \Omega} \leq \delta_{11}+\sqrt{\mu} \delta_{2}+d(\Omega)\right\| f^{p} \|_{0, \Omega}+8\left(|\Gamma|+\left\|g_{p}^{\epsilon}\right\|_{0, \Gamma}^{2}\right) .
$$

Using (3.28), estimate (4.6) becomes

$$
\left\|\nabla u_{\epsilon}^{p}\right\|_{0, \Omega} \leq \frac{1}{\sqrt{\mu}}\left(8 \delta_{6}^{2}+\delta_{11}+\sqrt{\mu} \delta_{2}+d(\Omega)|| f^{p} \|_{0, \Omega}+8\left(|\Gamma|+\|g\|_{0, \Gamma}^{2}\right)\right),
$$

and using (3.5) and (i) of (3.7), estimate (4.7) becomes

$$
\left\|\nabla u_{\epsilon}\right\|_{0, \Omega} \leq \frac{1}{\sqrt{\mu}}\left(\frac{1}{2}+8 \delta_{6}^{2}+\sqrt{\mu} \delta_{2}+\delta_{11}+d(\Omega)|| f \|_{0, \Omega}+8\left(|\Gamma|+\|g\|_{0, \Gamma}^{2}\right)\right) .
$$

By adequately choosing the positive numbers $\delta_{j}^{\prime} s$, we immediately get

$$
\left\|\nabla u_{\epsilon}\right\|_{0, \Omega} \leq \frac{1}{\sqrt{\mu}}\left[1+d(\Omega)\|f\|_{0, \Omega}+8\left(|\Gamma|+\|g\|_{0, \Gamma}^{2}\right)\right] .
$$

We conclude the theorem for $u$ by applying ( $a$ ) of Lemma 2.2 on one hand, and (iii) of Remark 2.2 on the other hand.

Finally; in order to get the explicit $H^{1}$ estimate of $u_{\epsilon}$, and so that of $u$, we use the Poincaré inequality (3.32) to bound $\|u\|_{0, \Omega}$ at one hand and the estimate (1.7) at the other hand.

\section{Conclusion}

In the point of view of numerical analysis, estimate of Theorem 1.1 is interesting. Indeed, error estimates in finite element method of the type

$$
\left\|u-u_{h}\right\|_{0, \Omega} \leq C h\|\nabla u\|_{0, \Omega}
$$

involve the quantity $\|\nabla u\|_{0, \Omega}$. Assuming that the constant $C$ can be explicitly computed, then it is possible to explicitly bound $\|\nabla u\|_{0, \Omega}$ which implies a better estimate of $\|u\|_{0, \Omega}$. 
Another interesting feature of the estimate (1.7) that makes it effective is that it does not depend on the characteristic parameters of the polygonal domain $\Omega$, namely, the edges's length, their number as well as the measures of the angles. The estimate is therefore indifferently applicable to all polygons. All this allows the possibility to generalize this result, by substantial approximation, to a $C^{1}$ class domain.

\section{Acknowledgments}

The authors would like to thank the referee for his relevant comments and remarks on the material presented in this paper as well as the editor for his support during the submission process.

\section{Appendix A. Some clarifications}

Regarding the application of the main result established in [7], we note the following facts. The part of Theorem 2.1 in [7] used in the proof of Lemma 3.2 is the equivalence between the following two assertions:

1) $X_{i}$ is approximated by smooth functions with support away from $\Gamma-\Gamma_{i}$;

2) $\lim _{r \rightarrow 0} \frac{1}{|B(x, r)|} \int_{B(x, r) \cap \Omega}\left|X_{i}(y)\right| \mathrm{d} y=0$.

To effectively apply this result, we need to show that the fact $X_{i}=0$ on $\Gamma-\Gamma_{i}$ implies 2). Indeed, let $x \in \Gamma-\Gamma_{i}$. We assume for convenience, that $x$ coincide with the origin $(0,0)$ of $\mathbb{R}^{2}$. Since $\partial \Omega$ is a polygon then, there exists $c>0$ independent of $r$ such that

$$
|B(0, r) \cap \Omega|=c|B(0, r)| .
$$

On the other hand, the function $X_{i}$ solution of problem $\left(P_{1}\right)$ is continuous on $\bar{\Omega}$. One can see this using the following argument: according to the definition of $u_{\epsilon}^{p}, \overline{X_{i}} \mid \partial \Omega \in C^{\infty}(\partial \Omega)$. Applying the Whitney extension theorem (see [12]), one can find $\overline{X_{i}} \in C^{\infty}(\bar{\Omega})$ that coincide with $X_{i}$ on $\Gamma$. Thus, $X_{i}-\overline{X_{i}} \in H^{1}(\Omega)$ solves the following problem

$$
\begin{cases}L\left(X_{i}-\overline{X_{i}}\right)=-L\left(\overline{X_{i}}\right) & \text { a.e in } \Omega, \\ X_{i}-\overline{X_{i}}=0 & \text { on } \cup_{i} \Gamma_{i} .\end{cases}
$$

Since the boundary $\Gamma$ of $\Omega$ satisfies the conditions (1.5) and $L\left(\overline{X_{i}}\right) \in L^{2}(\Omega)$ then, $X_{i}-\overline{X_{i}} \in$ $H^{\frac{3}{2}+\iota}(\Omega)$. Consequently

$$
X_{i}=\left(X_{i}-\overline{X_{i}}\right)+\overline{X_{i}} \in H^{\frac{3}{2}+\iota}(\Omega)
$$


as well. Furthermore, by an adequate Sobolev embedding, $X_{i} \in C^{0}(\bar{\Omega})$ and thus, the claimed continuity. The Lebesgue differentiation theorem yields:

$$
\begin{aligned}
& \lim _{r \rightarrow 0} \frac{1}{|B(x, r)|} \int_{B(x, r) \cap \Omega}\left|X_{i}(y)\right| \mathrm{d} y=\lim _{r \rightarrow 0} \frac{1}{|B(0, r)|} \int_{B(0, r) \cap \Omega}\left|X_{i}(y)\right| \mathrm{d} y \\
\leq & \lim _{r \rightarrow 0} \frac{1}{|B(0, r) \cap \Omega|} \int_{B(0, r) \cap \Omega}\left|X_{i}(y)\right| \mathrm{d} y=\left|X_{i}(0)\right|=0,
\end{aligned}
$$

we infer that assertion 2) holds for all $x \in \Gamma-\Gamma_{i}$ and thus, $C_{1,2}-$ almost everywhere. Where $C_{1,2}$ refers to the 2 -capacity of the set $\Gamma-\Gamma_{i}$.

Finally, one can show continuity of the function $Y_{i}$ that solves problem $P_{4}$ by remarking that $\sigma\left(Y_{i}\right) \cdot \vec{n}=g_{p}^{\epsilon} \in H^{\frac{1}{2}}\left(\Gamma-\Gamma_{i} \cup \Gamma_{0}\right)$ and by applying the same argument used for justifying the regularity of the solution $u$ of problem (1.1), one easily infer that $Y_{i} \in C^{0}(\bar{\Omega})$ and gets the same conclusions as for $X_{i}$.

\section{References}

[1] Duvaut G., Lions J. L., Les Inéquations en Mécanique et en Physique. Dunod, Paris, 1972.

[2] Nicaise S., About the Lamé system in a polygonal or polyhedral domain and a coupled problem between the Lamé system and the plate equation. I: regularity of the solutions. Ann. Sc. Norm. Super. Pisa Cl. Sci. (4)., 19 (3) (1992), 327-361.

[3] Allaire G., Analyse Numérique et Optimisation. Une introduction à la modélisation et à la simulation numérique. Editions de l'école Polytechnique, 2004.

[4] Adams R. A., Fournier J. J., Sobolev Spaces. Pure and applied mathematics series, 2nd ed, 2003.

[5] Horgan C. O., Korn's inequalities and their applications in continuum mechanics. SIAM Rev., 37 (4) (1995), 491-511.

[6] Di Nezzaa E., Palatucci G., Valdinoci E., Hitchhiker's guide to the fractional Sobolev spaces. Bull. Sci. Math., 136 (2012), 521-573.

[7] Egert M., Tolksdorf P., Characterizations of Sobolev Functions that vanish on a part of the boundary. Discrete Contin. Dyn. Syst. Ser. S, 10 (4) (2017), 729-743.

[8] Doktor P., Zenisek A., The density of infinitely differentiable functions in Sobolev spaces with mixed boundary conditions. Appl. Math., 51 (5) (2006), 517-547.

[9] Carstensen C., Sauter S. A., A posteriori error analysis for elliptic PDEs on domains with complicated structures. Numer. Math., 96 (4) (2004), 691-721.

[10] Repin S., Sauter S., Smolianski A., A posteriori error estimation for the Poisson equation with mixed Dirichlet-Neumann boundary conditions. J. Comput. Appl. Math., 164-165 (2004), 601-612.

[11] Eck C., Jarusek J., Krbec M., Unilateral Contact Problems: variational methods and existence theorems. Series: Chapman ans Hall/CRC Pure and Applied Mathematics, 2005.

[12] Whitney H., Analytic extensions of differentiable functions defined in closed sets. Trans. Amer. Math. Soc., 36 (1934), 63-89. 